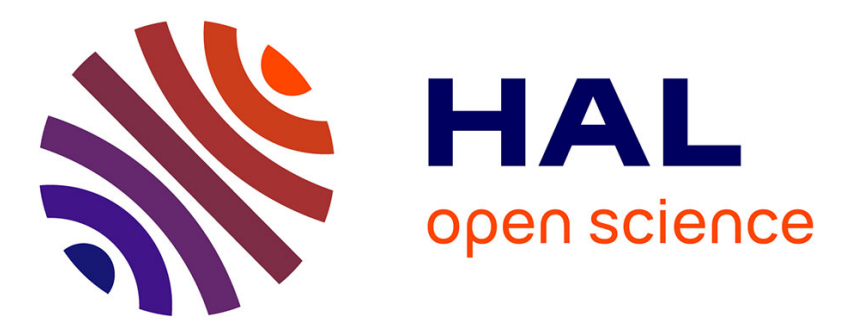

\title{
Large Reynolds number self-similar states of unstably stratified homogeneous turbulence
}

\author{
Alan Burlot, Benoît-Joseph Gréa, Fabien S. Godeferd, Claude Cambon, \\ Olivier Soulard
}

\section{- To cite this version:}

Alan Burlot, Benoît-Joseph Gréa, Fabien S. Godeferd, Claude Cambon, Olivier Soulard. Large Reynolds number self-similar states of unstably stratified homogeneous turbulence. Physics of Fluids, 2015, 27, pp.065114. 10.1063/1.4922817 . hal-01298326

\section{HAL Id: hal-01298326 \\ https://hal.science/hal-01298326}

Submitted on 7 Apr 2016

HAL is a multi-disciplinary open access archive for the deposit and dissemination of scientific research documents, whether they are published or not. The documents may come from teaching and research institutions in France or abroad, or from public or private research centers.
L'archive ouverte pluridisciplinaire HAL, est destinée au dépôt et à la diffusion de documents scientifiques de niveau recherche, publiés ou non, émanant des établissements d'enseignement et de recherche français ou étrangers, des laboratoires publics ou privés. 


\title{
Large Reynolds number self-similar states of unstably stratified homogeneous turbulence
}

\author{
A. Burlot, ${ }^{1,2, a)}$ B.-J. Gréa,,${ }^{1, b)}$ F. S. Godeferd, ${ }^{2}$ C. Cambon, ${ }^{2}$ and O. Soulard ${ }^{1}$ \\ ${ }^{1}$ CEA, DAM, DIF, F-91297 Arpajon, France \\ ${ }^{2}$ LMFA, Université de Lyon, École centrale de Lyon, CNRS, INSA, UCBL, \\ F-69134 Écully, France
}

(Received 23 April 2015; accepted 11 June 2015; published online 24 June 2015)

\begin{abstract}
We study the influence of the large scale energy distribution on the long term dynamics of unstably stratified homogeneous turbulence at high Reynolds number $R e=10^{6}$, using a statistical two-point spectral model based on the eddy-damped quasi-normal closure. We consider several initial spectral scalings $k^{s}$ in the infrared range with $s \in[1 ; 5]$ and we establish that the resulting kinetic energy growth rates are controlled by $s$, with the appearance of backscatter effects for $s \gtrsim 3.5$. We then assess that only for $s \leq 4$ do we observe self-similarity in the infrared and in the inertial ranges, but not in the dissipative range. Compensated energy and buoyancy spectra exhibit the expected Kolmogorov-Obukhov $k^{-5 / 3}$ scaling at long time, and a trend to the theoretically predicted $k^{-7 / 3}$ scaling for velocity-buoyancy cross-correlation spectrum thanks to the very large Reynolds number. We also show a direct link between the late-time anisotropy of the flows and the infrared spectrum, thus demonstrating long-lasting effect of initial conditions on unstably stratified turbulence. We show that, in addition to the Kolmogorov $k^{-5 / 3}$ scaling, the kinetic energy spectrum inertial range includes a $k^{-3}$ zone due to polarization anisotropy, and we confirm the clear $\sin ^{2} \theta$ dependence of the velocity-buoyancy spectrum in the inertial range, where $\theta$ is the orientation of the wave vector to the axis of gravity. However, an unexpected quick return to isotropy of the scalar spectra has been identified, which cannot be explained by a standard dimensional analysis. (C) 2015 AIP Publishing LLC. [http://dx.doi.org/10.1063/1.4922817]
\end{abstract}

\section{INTRODUCTION}

In variable-density flows submitted to gravity, configurations in which an unstable density stratification induces turbulent mixing are encountered in several occurrences of industrial, geophysical, or astrophysical flows. Energy production by inertial confinement fusion also enters this flow category. ${ }^{1}$ Modelling the complete evolution of the time-dependent inhomogeneous buoyancyinduced mixing from the onset of the relevant instabilities (e.g., the Rayleigh-Taylor (RT) instability) is rather complex as expressed by the variability of the mixing zone growth rates. ${ }^{2}$ In order to shed light on the different mechanisms at work in the flow, one may simplify the problem by considering unstably stratified homogeneous turbulence (USHT), wherein a continuous uniform density gradient feeds the flow dynamics. ${ }^{3-6}$ This idealized framework was previously studied experimentally by Thoroddsen et al..$^{7}$ by means of a heated grid. The inquiry of mixing by buoyancy-driven turbulence was first introduced by Batchelor $(1992)^{8}$ and, since then, several models where established. ${ }^{9} 10$ Considering the time evolution of flows from given initial conditions, the specific dynamics of USHT, with respect to other distorted turbulence dynamics-e.g., homogeneous isotropic turbulence (HIT), stably stratified turbulence (SST), and rotating turbulence $(\Omega \mathrm{T})$-resides in the

\footnotetext{
a)Electronic mail: alan.burlot@cea.fr

b)Electronic mail: benoit-joseph.grea@cea.fr
} 
growth of turbulent quantities due to the forcing of the mean density stratification. In the absence of additional sources terms, HIT, SST, or $\Omega$ T dynamics implies only a decay of energy, due to the nonlinear turbulent cascade and the dissipation.

The long time evolution of turbulent quantities in unstably stratified homogeneous turbulence leads to a self-similar regime. ${ }^{3}$ As in RT instability ${ }^{11,12}$ and homogeneous isotropic turbulence, ${ }^{13}$ this terminal regime allows to express the energy spectrum as $E(k, t)=u^{2}(t) \ell(t) \mathcal{G}(k \ell(t))$ with $u(t)$ a characteristic velocity, $\ell(t)$ a characteristic length scale, and $\mathcal{G}$ a dimensionless function. Multiple analyses were performed especially on HIT in self- or non-self-similar regimes (see, e.g., Meldi and Sagaut ${ }^{14}$ for an exhaustive review). A major result in both HIT and USHT is the role of large scale structures on the decay or growth of energy, and more specifically of large-scale dependence of two-point statistics, be it the velocity-correlation function $f(r \rightarrow \infty)$ or the low-wavenumber scaling of energy spectrum $E(k \rightarrow 0)$. For instance, one can assume the power law dependence $\lim _{k \rightarrow 0} E(k) \rightarrow k^{s}$ to leading order; in that case, the energy decay rate in HIT or growth rate in USHT can be estimated depending on $s$, which is commonly taken between 1 and 4 . Classical cases investigated are Batchelor turbulence with $s=4^{15}$ and Saffman turbulence with $s=2 \cdot{ }^{16,17}$ Both cases were also studied in turbulence submitted to rotation, ${ }^{18}$ stable stratification, or an externally applied magnetic field. ${ }^{19}$

In their recent analysis of freely decaying HIT experiments and modelling, Meldi and Sagaut ${ }^{14,20}$ discussed the availability of a long-time universal self-similar decay of energy with different scalings of the infrared (IR) spectral range-the energy spectrum range for wavenumbers $k$ from 0 up to the spectral peak (which is located approximately at the inverse of the integral length scale). Only the value $s=1$ seems to permit self-similar decay within all spectral ranges, and a second pivotal value $s=3.45$ appears, as in the work by Lesieur and Ossia, ${ }^{13}$ above which backscatter effects-a partial transfer of energy from inertial to large scales-do not allow the permanence of big eddies distribution.

In USHT, of course, no such decay is observed, and it is rather the growth rate which has to be investigated. From previous studies, it seems that it may depend on the initial distribution of large scales, but this was mainly assessed through the prism of amplified perturbations in a Rayleigh-Taylor mixing layer. As regards USHT with forced gravity and density gradient, recent studies ${ }^{4-6}$ conclude to an exponential self-similar growth of turbulent kinetic energy as $\sim e^{\beta N t}$; with $t$ the time, $N$ the buoyancy frequency characterizing the constant acceleration and mean stratification, and $\beta$ corresponding to the energy growth rate. Based on the arguments of Ref. 3, an explicit relation between $\beta$ and the IR power law $s$ was also proposed in Ref. 6 when $s \leq 4$ (or after a short transient when $s>4$, since then the infrared spectral range adjusts rapidly to $s=4$ ),

$$
\beta=\frac{4}{s+3} \text {. }
$$

This permits to link the essential role of large scales with the dynamics of USHT. However, in addition to the IR scaling, it appears that large-scale anisotropy is also very important, in that it accumulates energy at wavenumbers orthogonal to the axis of gravity, a spectral domain where buoyancy forcing acts linearly on the initial large eddies when $s<4$, but nonlinear interactions prevail when $s=4 .^{3}$

Accordingly, high Reynolds number unstably stratified turbulence evolving self-similarly is required to study the influence of big eddies distribution on the statistics of the flow. Simulations for USHT have been recently proposed, ${ }^{5}$ but there are limitations to the regimes direct numerical simulation (DNS) can reach. In USH or RT turbulence, not only the range of Reynolds numbers accessible with DNS is limited as in HIT, but also two other strong constraints apply: energy continuously grows due to the non zero vertical buoyancy flux, and a significant margin in the box size has to be preserved to accommodate the growth of the large scales, or, in other words, the infrared spectral range has to be wide enough. Altogether, the largest DNS realized to date (as, for instance, for $\mathrm{RT}^{21}$ ) reached a turbulent Reynolds number $R e \simeq 5000$, and were limited in time so that only a short similarity state period could eventually be accessed, and were obtained at a hefty computational price.

In HIT, significant computational power is required to reach turbulent regimes at relatively high Reynolds numbers. Statistical closures were successfully introduced in order to overcome this 
limitation. ${ }^{22,23} \mathrm{We}$, therefore, choose here also to tackle the problem at the level of the two-point statistics of turbulence, and hence use the eddy-damped quasi-normal Markovian (EDQNM) model for USH which has been developed and validated against DNS by Burlot et al. ${ }^{6}$ Two-point statistical EDQNM modelling was already successfully used to represent homogeneous isotropic ${ }^{24}$ and anisotropic turbulence in different contexts, ${ }^{25-27}$ and was recently introduced for USH turbulence. ${ }^{6}$ It demonstrated its ability to reproduce properly DNS results in a range of parameters (Reynolds number, time span) accessible to the latter, and to provide adequate results at very large Reynolds number and asymptotically long times. The model permits to consider various parameters: initial spectral distributions, kinetic energy to buoyancy variance ratios, and Froude numbers. It gives access to the full scale-dependent anisotropic two-point statistics in the axisymmetric representation.

Our goal in the present study is to provide further insight about the influence of the initial large scale structures that force the evolution of USHT. In the EDQNM model, this consists in varying the power law $k^{s}$ of the infrared scale in the initial energy spectra. We propose to use our spectral closure for USHT in order to explore large Reynolds number self-similar states and to explicitly investigate the link between the growth rate $\beta$ and the slope $s$ of the infrared spectra, extending ${ }^{3,5}$ to new parametric ranges. We pursue the analysis of self-similarity properties not only in the large scales but also in the inertial and the dissipative ranges to seek the existence of universal scaling laws. Therefore, the question raised is which scales of USH turbulence keep a memory of its generating initial conditions. In parallel, the anisotropy of the turbulent structures will be scrutinized in order to measure the influence of the buoyancy force.

The paper is organized as follows. First, we present the unstably stratified homogeneous turbulence configuration in Sec. II along with its governing equations, the EDQNM closure, and the initial conditions and parameters. Then, numerical results are discussed in Sec. III: non-dimensional numbers in Sec. III A, one-point statistics in Sec. III B, the time evolution of spectra in Sec. III C, and finally anisotropic characterization of velocity and buoyancy distributions in Sec. III D. Final comments and conclusions are provided in Sec. IV.

\section{UNSTABLY STRATIFIED HOMOGENEOUS TURBULENCE}

We first present in this section the dynamical equations for USHT written in terms of buoyancy, then we provide a quick description of the spectral closure model which is used to compute the long time statistics of the flow in a high Reynolds number self-similar regime. The reader can refer to Burlot et al. ${ }^{6}$ for a complete description.

\section{A. Dynamical equations for unstably stratified homogeneous turbulence}

We consider an incompressible flow in which turbulent quantities are assumed statistically homogeneous and axisymmetric about the vertical axis which bears gravity. The mean flow $\langle\mathbf{u}\rangle$ is assumed to be zero, whereas the fluid density has a uniform linear mean gradient in the vertical direction, which is assumed to be unchanged by density fluctuations. In addition, the contrast of density is supposed small allowing the Boussinesq approximation. The resulting buoyancy force is destabilizing when gravity and density gradients are of opposite sign, producing the unstably stratified situation. The following Navier-Stokes-Boussinesq equations are written for the divergenceless fluctuating velocity field $\mathbf{u}$ and for $\vartheta$, the fluctuating buoyancy rescaled as a velocity:

$$
\begin{array}{r}
\frac{\partial u_{i}}{\partial x_{i}}=0, \\
\frac{\partial u_{i}}{\partial t}+u_{j} \frac{\partial u_{i}}{\partial x_{j}}=-\frac{\partial p}{\partial x_{i}}+v \frac{\partial^{2} u_{i}}{\partial x_{j} \partial x_{j}}+N \vartheta \delta_{i 3}, \\
\frac{\partial \vartheta}{\partial t}+u_{j} \frac{\partial \vartheta}{\partial x_{j}}=\mathcal{D} \frac{\partial^{2} \vartheta}{\partial x_{j} \partial x_{j}}+N u_{3},
\end{array}
$$

where $p$ is the pressure divided by a reference density, $v$ the kinematic viscosity, and $\mathcal{D}$ the molecular diffusivity. The intensity of stratification is set by the buoyancy frequency $N$ by analogy with 
the Brunt-Väisälä frequency in the stably stratified case. The stratification time scale associated with the linear buoyancy term in Eq. (2b) is therefore $T_{N}=1 / N$.

For a flow periodic in all three directions, Eqs. (2a)-(2c) can be solved using classical pseudospectral discretization techniques, ${ }^{28}$ but such direct numerical simulations are computationally demanding. Moreover, in DNSs of stratified homogeneous turbulence, the velocity and buoyancy fields are post-processed to provide, e.g., two-point statistics such as energy spectra. We propose to use instead a model that solves equations for the two-point statistics. The outline of the corresponding spectral closure is described hereafter.

\section{B. Spectral closure}

An overview of the closure is given in this section. For a complete description, the reader may refer to the lecture given by Orszag ${ }^{24}$ for the isotropic case, to Cambon et al.,${ }^{26}$ Sagaut and Cambon $^{29}$ for the anisotropic axisymmetric configuration, and to Ref. 6 for the specific adaptation to unstably stratified homogeneous turbulence.

Instead of considering the velocity and buoyancy fields whose dynamics is ruled by Eqs. (2a)-(2c), one considers their two-point correlation functions, $\langle\boldsymbol{u}(\boldsymbol{x}) \boldsymbol{u}(\boldsymbol{x}+\boldsymbol{r})\rangle$ for velocity, $\langle\vartheta(\boldsymbol{x}) \vartheta(\boldsymbol{x}+$ $\boldsymbol{r})\rangle$ for buoyancy, and $\langle\boldsymbol{u}(\boldsymbol{x}) \vartheta(\boldsymbol{x}+\boldsymbol{r})\rangle$ for their cross-correlation. They do not depend on the location $\boldsymbol{x}$ in homogeneous turbulence, but indeed depend on the orientation of the separation vector $\boldsymbol{r}$ in anisotropic flows. Dynamical equations for these two-point statistics can be obtained from Eqs. (2a)-(2c), but are unclosed since they involve two-point triple correlations, as widely known. This issue related to the hierarchy of statistical moments is solved in the present work by using the eddy-damped quasi-normal Markovian closure which permits to express triple correlations in terms of second-order ones. The model provides closed equations for spectra of the above-mentioned correlation functions, transforming the dependence on the separation vector $r$ in physical space into a dependence on the wave vector $\boldsymbol{k}$ in the Fourier representation. We shall use the following spectra arising from the two-point correlations statistics.

- The Reynolds stress tensor spectrum $\mathcal{R}_{i j}(\boldsymbol{k})$ such that $\mathcal{R}_{i j}(\boldsymbol{k}) \delta\left(\boldsymbol{k}+\boldsymbol{k}^{\prime}\right)=\left\langle\hat{u}_{i}(\boldsymbol{k}) \hat{u}_{j}\left(\boldsymbol{k}^{\prime}\right)\right\rangle$ where $\hat{u}_{i}$ denotes the Fourier coefficient of velocity component $u_{i}$ and $\delta$ being the 3D Dirac function.

- From that the kinetic energy spectrum can be defined as $\mathcal{E}(\boldsymbol{k})=\mathcal{R}_{i i}(\boldsymbol{k}) / 2$ assuming summation over the repeated index $i$.

- The buoyancy spectrum $\mathcal{B}(\boldsymbol{k})$ with $\mathcal{B}(\boldsymbol{k}) \delta\left(\boldsymbol{k}+\boldsymbol{k}^{\prime}\right)=\left\langle\hat{\vartheta}(\boldsymbol{k}) \hat{\vartheta}\left(\boldsymbol{k}^{\prime}\right)\right\rangle$ with similarly $\hat{\vartheta}$ the Fourier coefficient of $\vartheta$.

- The co-spectrum of vertical velocity-buoyancy two-point correlation, $\mathcal{F}(\boldsymbol{k})$, which is defined from $\mathcal{F}(\boldsymbol{k}) \delta\left(\boldsymbol{k}+\boldsymbol{k}^{\prime}\right)=\left\langle\hat{u}_{3}(\boldsymbol{k}) \hat{\vartheta}\left(\boldsymbol{k}^{\prime}\right)\right\rangle$.

In order to obtain the EDQNM closed equations for $\mathcal{R}_{i j}, \mathcal{E}, \mathcal{F}, \mathcal{B}$, Eqs. (2a)-(2c) are Fourier transformed. This helps to eliminate pressure by using incompressibility stated in Fourier space: $\boldsymbol{k} \cdot \hat{\boldsymbol{u}}=0$. The dynamical equations for the two-point spectra are obtained by multiplication by $\hat{\boldsymbol{u}}(\boldsymbol{k})$ and $\hat{\vartheta}(\boldsymbol{k})$ and taking the ensemble average \langle\rangle . The classical turbulence problem of the hierarchy of dynamical equations occurs then, with second-order moments involving third-order moments. The core idea of the EDQNM closure is to assume that third-order moments are linearly relaxed by fourth-order cumulants, which are the departure of the fourth-order statistics from that of a Gaussian distribution. Formally, this provides an expression of the third-order moment involving only products of second-order moments, and hence a set of closed dynamical equations for second-order statistics, namely, the desired spectra.

In USH turbulence, in addition to the quasi-normal assumption, one introduces a second hypothesis on the third-order moments whose dynamics is enriched by explicit buoyancy and stratification terms in the dynamical equations. These terms, which are ignored in Ref. 30, are explicitly accounted in Ref. 31 for the stable stratification case. Here, we use the simple assumption that nonlinear contributions of stratification-related terms in the dynamics of third-order moments can be replaced by an additional damping to nonlinear terms following the successful procedure described in Ref. 6 and supported by DNS results. 
In the end, one obtains a set of coupled integro-differential equations whose discretization yields the evolution in time of the spectra for $N_{k}$ discretized wavenumbers $k$ in the range $\left[k_{m}, k_{M}\right]$, and for $N_{\theta}$ polar orientations $\theta$ of the corresponding wave vector $\boldsymbol{k}$ about the axis of gravity. For instance, $\theta=0$ corresponds to motion perpendicular to the axis of gravity $(\boldsymbol{k}$ being aligned with gravity, and from incompressibility $\boldsymbol{k} \cdot \hat{\boldsymbol{u}}=0$ ). Axisymmetry permits to analytically integrate the spectra over the azimuthal angle. The output of EDQNM computations is therefore a set of $N_{k} \times N_{\theta}$ discrete values of velocity, buoyancy, and flux spectra distributed in Fourier space with a polar-spherical mesh.

All the following computations were done using $N_{k}=128$ discretized wavenumbers $k$ between $k_{m}=10^{-2}$ and $k_{M}=10^{4}$ and $N_{\theta}=21$ polar angles. The logarithmic distribution of $k$ s therefore allows for $\sim 20$ wavenumbers per decade, which is well above the already comfortable value of 10 wavenumbers per decade recommended for isotropic EDQNM resolution. We checked wavenumber convergence using 512 and 1024 points in order to evaluate if long range interactions could modify the solution, without observing any noticeable change. No further dependence is observed when varying the angular discretization.

It is convenient to work with spherically integrated spectra obtained by integration of $\boldsymbol{k}$ or $(k, \theta)$-dependent spectra $\mathcal{R}_{i j}(k, \theta), \mathcal{E}(k, \theta), \mathcal{B}(k, \theta)$, and $\mathcal{F}(k, \theta)$ over all orientations of $\boldsymbol{k}$. In doing so, one only retains a global effect of anisotropy at each scale corresponding to a given wavenumber $k$ (again, the model is capable of full axisymmetric description). We shall denote $R_{i j}(k), E(k), B(k)$, and $F(k)$ the corresponding spherically integrated spectra (for instance $E(k)=$ $\left.2 \pi k^{2} \int_{0}^{\pi} \mathcal{E}(k, \theta) \sin \theta \mathrm{d} \theta\right)$.

\section{Varying the initial distribution of big eddies}

As previously done in isotropic turbulence using the EDQNM closure, ${ }^{13}$ we investigate the evolution of USHT starting with different distributions of the big eddies, that is, we study the influence of the large scales spectral distribution on energy growth rate and flow anisotropy. With respect to isotropic turbulence, two additional parameters need to be introduced: the initial ratio of buoyancy variance to kinetic energy, as a trace of the way stratification is introduced in the flow (for instance, in heated grid turbulence the corresponding initial buoyancy variance is very small ${ }^{7}$ ); and the Froude number that quantifies the intensity of stratification with respect to inertial effects.

We tune these parameters using the analytical spectral distribution for kinetic energy ${ }^{32}$

$$
E(k, t=0)=A_{s}\left(\frac{k}{k_{\text {peak }}}\right)^{s} \exp \left(-\frac{s}{2}\left(\frac{k}{k_{\text {peak }}}\right)^{2}\right),
$$

where $k$ is the wavenumber, $k_{\text {peak }}$ is the most energetic wavenumber, $s$ is the slope of the infrared spectra, and $A_{s}$ sets the total initial kinetic energy $\mathcal{K}(t)=\int_{0}^{+\infty} E(k, t) \mathrm{d} k$. Along with a choice of kinematic viscosity $v=5 \times 10^{-4}$, the kinetic energy is used to compute the initial Reynolds number as $R e=\mathcal{K}^{2} /(\varepsilon v)$, where kinetic energy dissipation is $\varepsilon(t)=2 v \int_{0}^{+\infty} k^{2} E(k, t) \mathrm{d} k$. Spectra as in Eq. (3) are used to initialize the buoyancy spectrum $B(k, t=0)$, and the initial flux is $F(k, t=0)=0$.

The Froude number is then obtained as $F r=\varepsilon /(\mathcal{K} N)$ where $N$ is the buoyancy frequency. The larger $F r$, the more important the intensity of stratification, and thus the buoyancy force. The third non-dimensional parameter is $\Lambda=\langle\vartheta \vartheta\rangle / \mathcal{K}$ which we choose to be $\Lambda=1$ at the beginning of all the simulations. Here, the buoyancy variance is related to its spectrum as $\langle\vartheta \vartheta\rangle(t)=\int_{0}^{+\infty} B(k, t) \mathrm{d} k$. We have checked that the precise value of $\Lambda$ has no influence on the asymptotic similarity state, only on the transient short-time growth. The initial parameters for the computations are as follows: the Reynolds number is $R e=833$ and the Froude number is $F r=1.2$. The corresponding spectral peaks for each value of the infrared power law $k^{s}$ with $s$ from 1 to 5 are detailed in Table I. The corresponding initial spectra are shown as a subset of curves in Figs. 3(a) and 3(b). From these initial narrow-band spectra, turbulence develops an inertial range and a dissipative one, with or without adjustment of the infrared range energy distribution. As observed in Figure 3, the infrared range spans almost four decades, so that the large scale cutoff has negligible effect, unlike what may be observed in DNS at long time, even at high resolution. 
TABLE I. Peak wavenumbers for each power law $s$ chosen in our nine simulations, and non dimensional final time $N t$, at which $R e=10^{6}$.

\begin{tabular}{lccccccccc}
\hline \hline$s$ & 1 & 1.5 & 2 & 2.5 & 3 & 3.5 & 4 & 4.5 & 5 \\
\hline$k_{\text {peak }}$ & 34.64 & 37.95 & 40 & 41.4 & 42.43 & 43.2 & 43.82 & 44.32 & 44.72 \\
$N t$ & 9.4 & 10.4 & 11.9 & 13.1 & 14.5 & 16.2 & 16.9 & 17.4 & 17.6 \\
\hline \hline
\end{tabular}

Finally, the computations are carried out until $R e=10^{6}$ for each case, corresponding to a different final time $N t$ as shown in Table I.

\section{NUMERICAL RESULTS}

We analyse here the parametric influence of the IR power law $s$ on the dynamics of USH turbulence, in particular on one-point statistics which are obtained by integrating the spectra over the complete spectral space. In Sec. III A, we first discuss the flow regime characterized by Reynolds and Froude numbers, and by the mixing parameter $\Lambda$, then the evolution of kinetic energy, buoyancy and vertical flux variances in Sec. III B. Second, we shall discuss in Sec. III C the observed similarity in the time-dependent spectra, and the evolution of inertial and infrared ranges.

\section{A. Flow regime characterized by $R e, F r$, and $\Lambda$}

The time evolution of the three non-dimensional parameters characterizing the flow is shown in Figure 1 in terms of non-dimensional time $t^{*}=N t$.

First, the transient phase is similar to the one described in Ref. 6: at short time, the flow adjusts in the small scales and the Reynolds and Froude numbers decrease, as a result of the transfer of kinetic energy from large to small dissipative scales. The transient decrease stops after about 2 stratification periods, and a monotonic asymptotic behaviour is observed, starting at $t^{*} \simeq 5$.

The Reynolds number (Figure 1(a)) reaches a long-time exponential behaviour, evolving more rapidly for low values of $s$ than for larger ones. All runs start from $R e=833$ to finally reach $R e \simeq 10^{6}$. (Note that due to the exponential growth, computational time becomes long due to the extremely low time step $\delta t^{*} \sim 10^{-7}$.) The Reynolds number growth depends on the initial conditions: the shallower the spectrum, the sooner the self-similar state is reached. Self-similarity starts uniformly around $R e=2 \times 10^{3}$ but occurs from $t^{*} \simeq 3$ for $s=1$ to $t^{*} \simeq 6$ for $s=5$. We notice the same exponential growth for $s \geq 4$. This is correlated to the evolution of kinetic energy whose growth rate is bounded by the infrared slope $s=4$ due to backscatter effects (see below).

In all cases, the Froude number (Figure 1(b)) which starts above unity ( $F r=1.2$ for all runs), corresponding to weakly stratified flow, eventually reaches an asymptotic value lower than unity, showing that the flow dynamics is therefore significantly altered by stratification. This attests of the equilibrium between buoyant and inertial forces. Final values are spread between $F r=0.3$ for $s=1$ and $F r=0.48$ for $s \geq 4$. This asymptote of Froude number indicates the grip of stratification effects
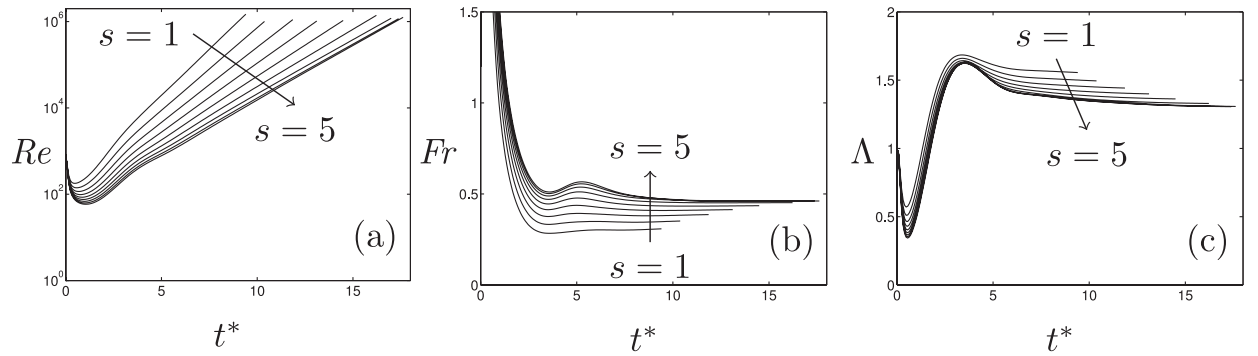

FIG. 1. Non-dimensional parameters evolution: (a) Reynolds number $\operatorname{Re}=\mathcal{K}^{2} /(v \varepsilon)$; (b) Froude number $F r=\varepsilon /(N \mathcal{K})$; (c) mixing parameter $\Lambda=\langle\vartheta \vartheta\rangle / \mathcal{K}$. 
on the flow which is stronger when the infrared slope is lower. It is explained by the energy of large scales structures which is more important for $s=1$ than $s=4$, is less submitted to non-linear transfer than linear buoyancy force. Furthermore, we observe that the maximum asymptotic Froude number is reached for $s=4$ where $F r=0.48$. Increasing $s$ further does not seem to produce a decrease of the asymptotic Froude number.

Finally, the time evolution of the mixing parameter $\Lambda$ is presented in Figure 1(c). All runs start with $\Lambda=1$, meaning there is exact equipartition between buoyancy variance and kinetic energy. The short-time depletion of $\Lambda$ is expected as in stably stratified turbulence, because the cascade of scalar is more efficient than the turbulent energy cascade. Later, one expects a self-similar mixing regime characterized by a plateau of $\Lambda$. However, Figure 1(c) shows that after reaching a peak value, at long time $\Lambda$ evolves slowly, but nonetheless decays. The asymptotic value is rather concentrated, between $\Lambda=1.6$ for $s=1$ and $\Lambda=1.3$ for $s=5$. This means that the flow contains $30 \%-60 \%$ more buoyancy variance than kinetic energy in its asymptotic regime. Here again, the large-scale distribution with a slope $s=4$ seems to mark a limit of mixing efficiency in USH turbulence.

\section{B. Energy and flux self-similar growth}

Figure 2 shows the time evolution of kinetic energy $\mathcal{K}$, buoyancy variance, and vertical buoyancy flux $\left\langle u_{3} \vartheta\right\rangle=\int_{0}^{+\infty} F(k) \mathrm{d} k$. The exponential growth $e^{\beta N t}$, with $\beta$ defined by Eq. (1), expected in the self-similar regime, is plotted for each case with dashed lines. Clearly, all our simulations exhibit the self-similar regime for kinetic energy and buoyancy variance evolution, and for the flux. For these three quantities, the asymptotic growth is fastest for the lowest infrared slope $s=1$, and decreases with increasing $s$. A saturation of this increase is observed at large $s$ above $s \simeq 4$, say, which is explained by backscatter effects observed in the energy and transfer spectra in Figures 3(a) and 4, discussed in Sec. III C.

We compare in Table II the theoretically predicted asymptotic growth with the measured one from the time evolution of kinetic energy $\mathcal{K}(t)$, buoyancy variance $\langle\vartheta \vartheta\rangle(t)$, and vertical buoyancy flux $\left\langle u_{3} \vartheta\right\rangle(t)$. For all three quantities, the growth rate is in very good agreement with the theoretical value for all cases of $s \in[1 ; 4]$. This also demonstrates that sufficiently long computations are needed to recover the right exponent, which should not be measured earlier than $N t \simeq 6$.

\section{Detailed spectral adjustments}

Our aim in this section is to discuss the turbulent dynamics from the point of view of the large scales - the infrared range - on the one hand, and of the inertial range, on the other. These are discussed in the respective Secs. III C 1 and III C 2, where similarity formulas are applied to expose each range.

The spherically integrated spectra $E(k), B(k)$, and $F(k)$ are plotted in Figures 3(a), 3(b), and $3(c)$, respectively. It shows the initial time distribution of kinetic energy and buoyancy (initial flux is zero), as well as their state when the flow reaches a very high Reynolds number $R e=10^{6}$. It can be seen at first sight that the energy and the buoyancy spectra evolve to a similar Kolmogorov-Obukhov
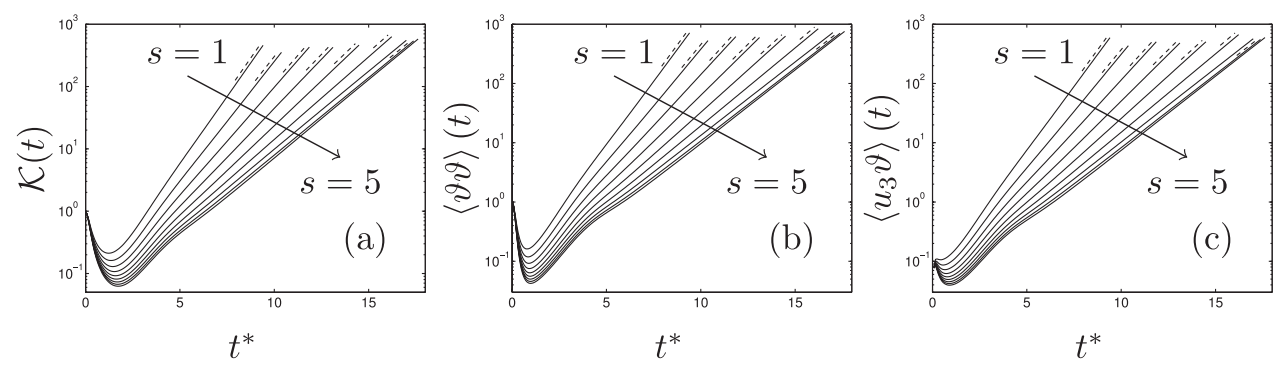

FIG. 2. (a) Kinetic energy, (b) buoyancy variance, and (c) vertical buoyancy flux evolution with slope of self-similar regime. Dashed lines represent the theoretical self-similar dynamics based on the formula $\mathcal{K}(t) \sim \exp (\beta N t)$ with $\beta$ given by Eq. (1). 
TABLE II. Values of $\beta$ at the end of each computation for the kinetic energy, the buoyancy variance, and the scalar flux compared to the theoretically predicted one from Eq. (1), $\beta_{t h}$, which is identical for the three quantities. The different instantaneous growth rates are obtained in the simulations from $\beta^{X}=\frac{1}{X} \frac{d X}{d N t}$.

\begin{tabular}{lccccccc}
\hline \hline $\mathrm{s}$ & $\beta_{\mathrm{th}}$ & $\beta_{\mathrm{sim}}^{\mathcal{K}}$ & $\beta_{\text {sim }}^{\mathcal{K}} / \beta_{\mathrm{th}}(\%)$ & $\beta_{\text {sim }}^{\langle\vartheta \vartheta\rangle}$ & $\beta_{\text {sim }}^{\langle\vartheta \vartheta\rangle} / \beta_{\mathrm{th}}(\%)$ & $\beta_{\text {sim }}^{\left\langle u_{3} \vartheta\right\rangle}$ & $\beta_{\text {sim }}^{\left\langle u_{3} \vartheta\right\rangle} / \beta_{\mathrm{th}}(\%)$ \\
\hline 1 & $4 /(1+3)$ & 0.993 & 99.3 & 0.989 & 98.9 & 0.994 & 99.4 \\
1.5 & $4 /(1.5+3)$ & 0.885 & 99.5 & 0.881 & 99.2 & 0.886 & 99.7 \\
2 & $4 /(2+3)$ & 0.797 & 99.6 & 0.794 & 99.3 & 0.798 & 99.7 \\
2.5 & $4 /(2.5+3)$ & 0.724 & 99.6 & 0.722 & 99.3 & 0.725 & 99.7 \\
3 & $4 /(3+3)$ & 0.663 & 99.5 & 0.661 & 99.2 & 0.664 & 99.6 \\
3.5 & $4 /(3.5+3)$ & 0.610 & 99.1 & 0.609 & 98.9 & 0.610 & 99.2 \\
4 & $4 /(4+3)$ & 0.572 & 100.2 & 0.571 & 99.9 & 0.572 & 100.1 \\
4.5 & $4 /(4+3)$ & 0.570 & 99.7 & 0.568 & 99.4 & 0.570 & 99.7 \\
5 & $4 /(4+3)$ & 0.573 & 100.3 & 0.571 & 100.0 & 0.573 & 100.2 \\
\hline \hline
\end{tabular}

$k^{-5 / 3}$ scaling, while the buoyancy flux follows roughly a $k^{-7 / 3}$ scaling law in the inertial range. The dissipative ranges are also very similar, but by contrast the infrared range that feeds the energy cascade is much different. Indeed, among the initial 9 different values chosen for $s$, the infrared distributions of spectra at $s=4,4.5$, and 5 rejoin to a single $k^{4}$ distribution in the large scales. The infrared zones of the spectra at $s=4.5$ and 5 have therefore evolved to Batchelor-type infrared spectra with $s=4$. The well-known backscatter effects rearrange in a few eddy turnover times the energy distribution at large scale. ${ }^{33}$ This effect can be more visible on large-scale compensated spectra in Sec. III C 1, but can also be demonstrated by examining the kinetic energy transfer spectra of Figure 4, that is, the transfer spectra $T(k)$ for the spherically integrated spectrum $E(k)$ (whose dynamics would follow a Lin type equation). ${ }^{34}$ The figure shows that, even in this highly anisotropic flow, energy transfer in USH turbulence consists of a strong downscale transfer with negative transfer in the large scales and positive transfer in the small ones. Both regions are separated by a well-defined inertial range in which the energy flux is constant, due to the very high Reynolds number regime. However, when closing up on the very large scale region, at smaller wavenumbers, the inset in Figure 4 shows the existence of backscatter of energy in the large scales for $s>2$, although of small amplitude compared with the forward cascade. The observed threshold $s=2$ corresponding to the dominance of backscatter term in the transfer is coherent with distant interaction expansion of the EDQNM closure shown by Ref. 33. The backscatter is largest for $s \geq 4$ and explains the rapid recovery of Batchelor scaling $k^{4}$ if the turbulent infrared range initially scales as $k^{s}$ with $s>4$. In addition, the importance of distant interaction between modes for backscatter dominated transfers can be verified by $T(k) \sim k^{4}$ for $k \rightarrow 0$ in Figure 4 as already mentioned in Lesieur $^{33}$ and Soulard et al. ${ }^{3}$
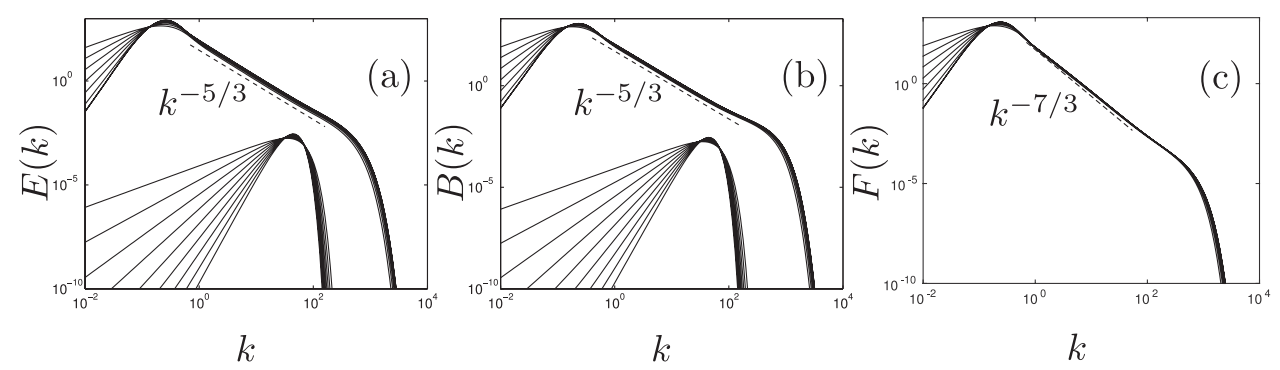

FIG. 3. Initial spectra (bottom set of curves in each panel, divided by 10) and spectra (top set) at $\operatorname{Re}=10^{6}$ of: (a) kinetic energy $E(k)$; (b) buoyancy variance $B(k)$; and (c) buoyancy-velocity cross-correlation $F(k)$. Each set of curves represents simulations with $s$ from 1 to 5 . The straight lines indicate the inertial range scaling $k^{-5 / 3}$ for $E(k)$ and $B(k)$ and $k^{-7 / 3}$ for $F(k)$. 

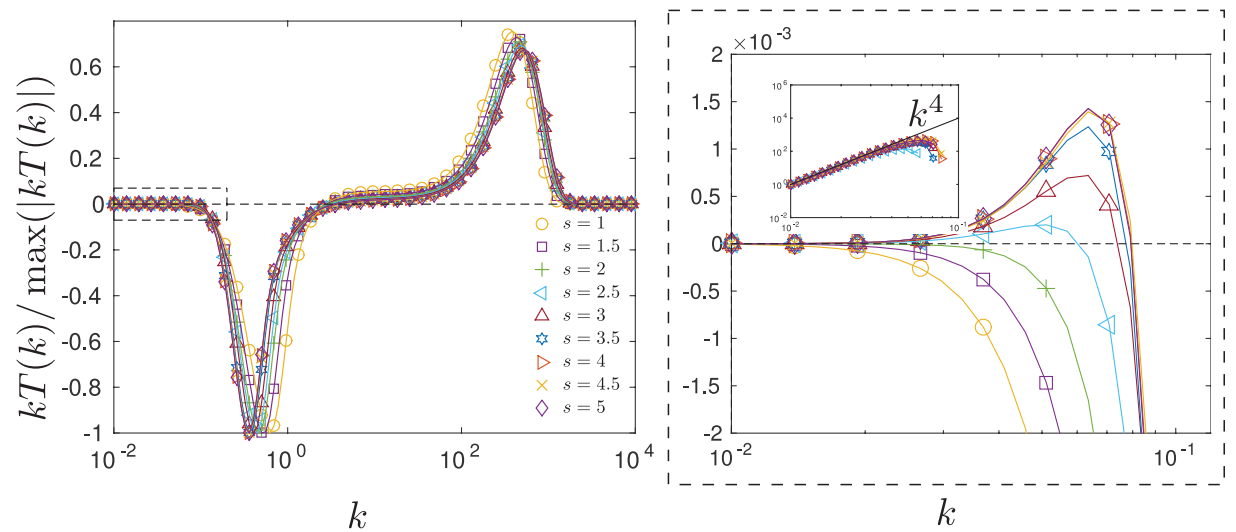

FIG. 4. Left: $k$-weighted kinetic energy transfer spectrum normalized by its maximum value $k T(k) / \max (|k T(k)|)$ for the different cases at $s=1$ to 5 and $\operatorname{Re}=10^{6}$. (The area beneath the curves is proportional to the transfer in this lin-log representation.) Right : A close-up of the rectangle shown with dashed lines in the small $k$ region. Inset: $\log -\log$ representation of renormalized transfers $T(k) / T\left(k_{m}\right)$ showing typical $k^{4}$ scaling for $s>2$ due to backscatter effects.

\section{Large scale adjustment in the infrared spectral range}

Figures 5 and 6, respectively, show the large-scale compensated spectra for the kinetic energy $E(k)$ on the one hand, and for the buoyancy variance $B(k)$ and the vertical buoyancy flux $F(k)$ on the other. The rescaling formula is

$$
X_{\text {infrared }}^{*}(k, t)=\frac{X(k, t)}{X\left(k_{m}, t\right)} \times\left(\frac{k}{k_{m}}\right)^{-s},
$$

where $X$ is replaced by $E, B$, and $F$ successively, and $k_{m}$ is the smallest wavenumber of the simulation. Equation (4) is an adaptation of the classical formula used in isotropic turbulence. ${ }^{13,35}$ By applying rescaling (4), one compensates the growth of energy or flux (the division by $X\left(k_{m}, t\right)$ ) and transforms to a plateau the infrared range assumed to scale as $k^{s}$ at each time. Departure from horizontal means a departure of the infrared spectral range from its initial distribution.

For ease of presentation, kinetic energy spectra in Figure 5 are plotted only for runs using $s=1$, $2,3,3.5,4$, and 5 , in semi-log scale.
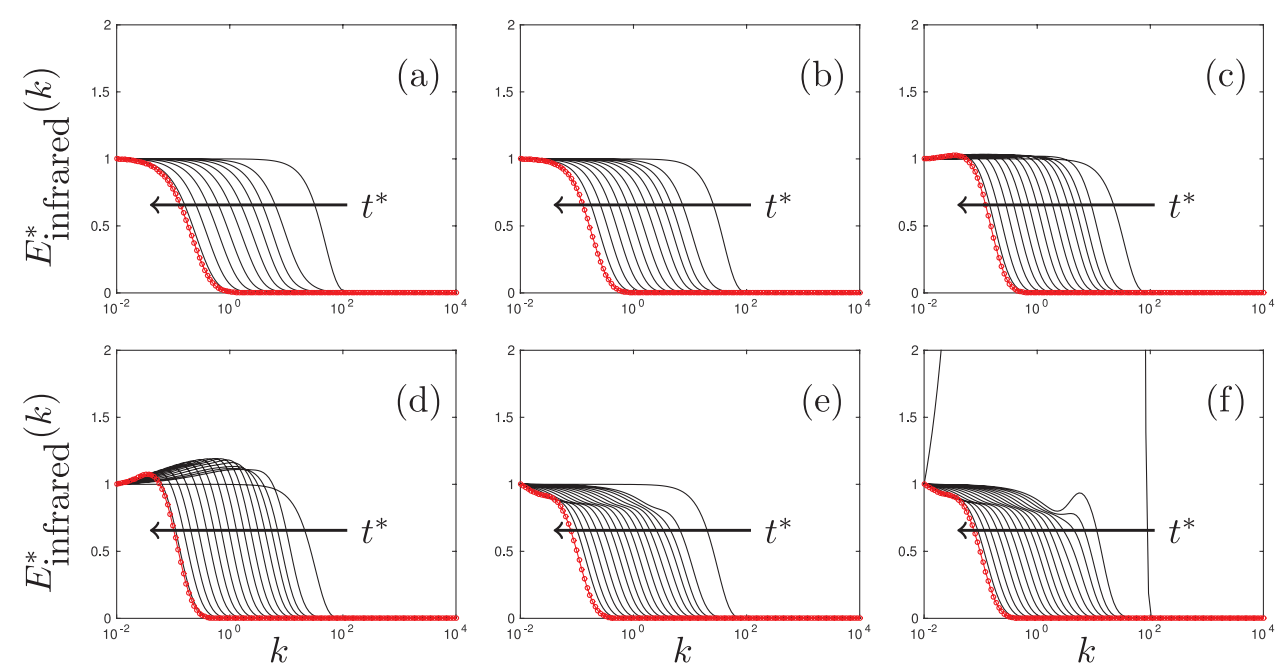

FIG. 5. Large-scale spectrum of kinetic energy $E(k)$ compensated using Eq. (4) at integer intermediate times from $t^{*}=0$ to the last time given in Table I. Increasing times as indicated by the arrows. The last computed spectrum is indicated by symbols (red color online) and corresponds to different times given hereafter for the different cases: (a) $s=1$ (last time $t^{*}=9.4$ ); (b) $s=2\left(t^{*}=11.9\right)$; (c) $s=3\left(t^{*}=14.5\right)$; (d) $s=3.5\left(t^{*}=16.2\right)$; (e) $s=4\left(t^{*}=16.9\right)$; and (f) $s=5\left(t^{*}=17.6\right)$. (Note that in panel (f), the initial spectrum at $t^{*}=0$ is initially $k^{5}$ and compensated by $\sim k^{-4}$, so that it gets out of the figure, but quickly recovers $k^{4}$ within half a stratification period.) 

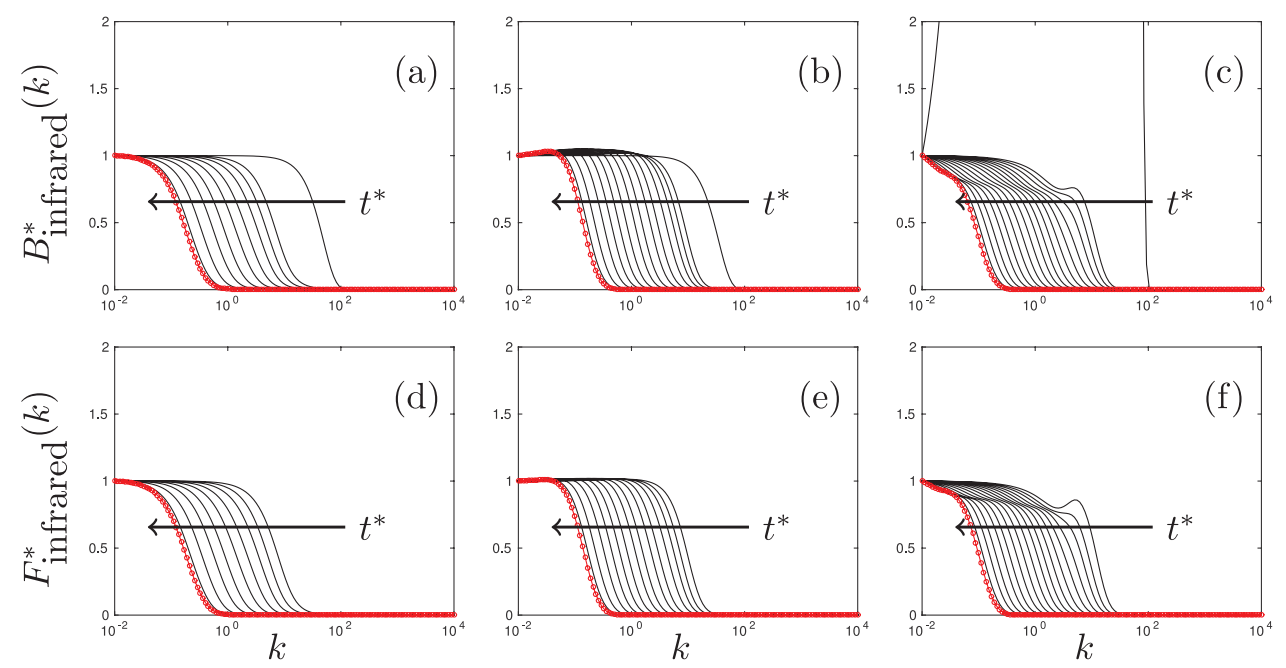

FIG. 6. Large-scale spectrum of buoyancy variance $B(k)$ compensated using Eq. (4) at the same times $t^{*}$ as in Figure 5, for: (a) $s=1$; (b) $s=3$; (c) $s=5$. And the compensated spectrum $F(k)$ of velocity-buoyancy correlation for: (d) $s=1$; (e) $s=3$; (f) $s=5$. (Note that, as in Figure 5(f), for panel (c) the initial curve for $B(k)$ gets out of the plot for the same reason; moreover, the initial fluxes at $t^{*}=0$ in panels (d)-(f) are initially zero.)

In Figures 5(a) and 5(b), for $s=1$ and 2, the compensated kinetic energy spectrum exhibits a plateau in the first two decades of $k$ at all times, so that the infrared energy scaling does not change at all. For $s=3$ in Figure 5(c), one observes that the infrared slope begins to depart slightly from the initial $k^{3}$ dependence, and for $s=3.5$ in Figure 5(d), it is clear that the similarity of large scales is not valid anymore since $s$ increases towards 4 , unlike cases $s=4$ (Batchelor turbulence) and 5 in Figures 5(e) and 5(f), where the infrared slope decreases slightly. Finally, for $s=5$, we observe a quick reshape of the spectra in the region connecting infrared and inertial ranges - the observed bump near $k \sim 50$ in the early times in Figure 5(f)—such that the spectrum eventually recovers an almost identical evolution as for the case $s=4$ due to backscatter. In a study of isotropic turbulence using the EDQNM model, Lesieur and Ossia find a limit $s=3.45$ above which backscatter appears and the infrared scaling evolves in time. ${ }^{13}$ This is consistent with what we observe here in USH turbulence.

The same comments apply for the evolution of the buoyancy variance spectrum $B(k)$ and the vertical buoyancy flux $F(k)$, plotted, respectively, in Figures 6(a)-6(c) and 6(d)-6(f) only for cases $s=1,3$, and 5 for the sake of brevity. This denotes that the dynamics of kinetic energy and buoyancy variance are closely intertwined.

To summarize, the EDQNM simulations allow to assess the importance of backscatter in the growth of turbulent quantities of USHT. The predictions established by Eq. (1) assume negligible backscatter effects and accordingly are valid for $s \leq 4$. However, backscatter still has an influence around $s=3.5$ by slowly modifying the similar distribution of energy at large scales although dominated by buoyancy production. Observe that the predictions given by Eq. (1) in Table II slightly overestimate the growth rates particularly around $s=3.5$. This tendency can be interpreted as evidence of backscatter influence.

\section{Inertial range scaling}

We focus here on the inertial range in the spectra, by renormalizing them as for classical Kolmogorov-Obukhov turbulence, using the kinetic energy dissipation $\varepsilon(t)$ and buoyancy dissipation $\varepsilon_{B}(t)$,

$$
\begin{array}{r}
E_{\text {inertial }}^{*}(k, t)=E(k, t) \times \varepsilon(t)^{-2 / 3} k^{5 / 3}, \\
B_{\text {inertial }}^{*}(k, t)=B(k, t) \times \varepsilon_{B}(t)^{-1} \varepsilon(t)^{1 / 3} k^{5 / 3}, \\
F_{\text {inertial }}^{*}(k, t)=F(k, t) N^{-1}\left(C_{K} \varepsilon(t)^{1 / 3}+C_{O} \varepsilon_{B}(t) \varepsilon(t)^{-2 / 3}\right)^{-1} k^{7 / 3},
\end{array}
$$



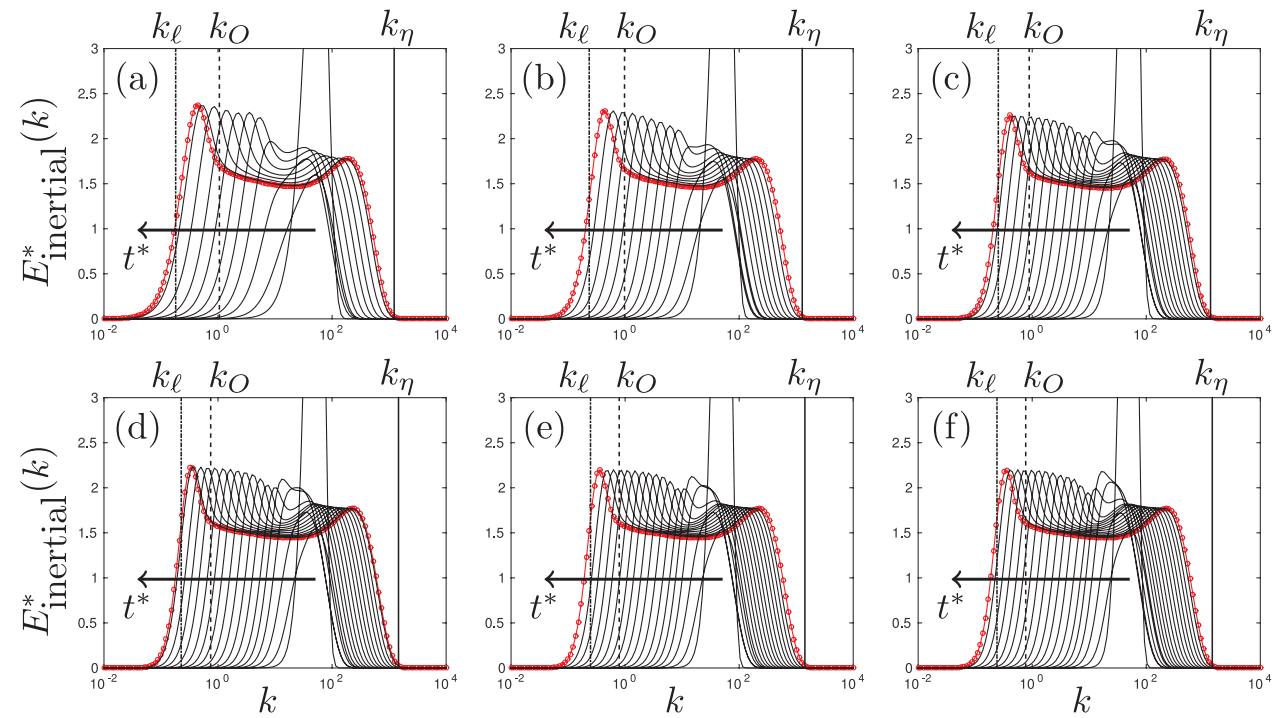

FIG. 7. Inertial range compensated spectra from Eq. (5a) for kinetic energy $E(k$ ): (a) $s=1$; (b) $s=2$; (c) $s=3$; (d) $s=3.5$; (e) $s=4$; and (f) $s=5$. Times are as in Figure 5, and the latest spectrum is denoted with symbols (red color online). (Note that the initial narrow-banded spectra get out of the plots but are indicated for better appreciation of the time evolution.) Vertical lines locate the wavenumbers $k_{\ell}, k_{O}$, and $k_{\eta}$ corresponding, respectively, to the integral length scale, to the Ozmidov scale and to the Kolmogorov scale for this last spectrum.

with the classical inertial power law $-5 / 3$ for the kinetic energy spectrum $E(k)$ and for the buoyancy spectrum $B(k)$ as suggested by dimensional analysis. ${ }^{36,37}$ We also choose $-7 / 3$ for the buoyancyvelocity co-spectrum $F(k)$, following experimental measurements in grid turbulence ${ }^{38}$ and theoretical predictions of Ref. 39. The dependence on dissipation in Eq. (5c) is obtained by assuming equilibrium between non linear terms and production in the equation for the vertical buoyancy flux spectrum as in Ref. 40. Here, $C_{K}$ and $C_{O}$ correspond, respectively, to the Kolmogorov and Obukhov constants. Figure 7 shows these compensated spectra for $E(k)$, and Figure 8 for $B(k)$ and $F(k)$.

Figures 7(a)-7(f) show that the rescaled kinetic energy spectrum $E_{\text {inertial }}^{*}(k)$ evolves similarly for all infrared power laws $s$. On these plots, the rescaled initial spectra (curves that do not fit
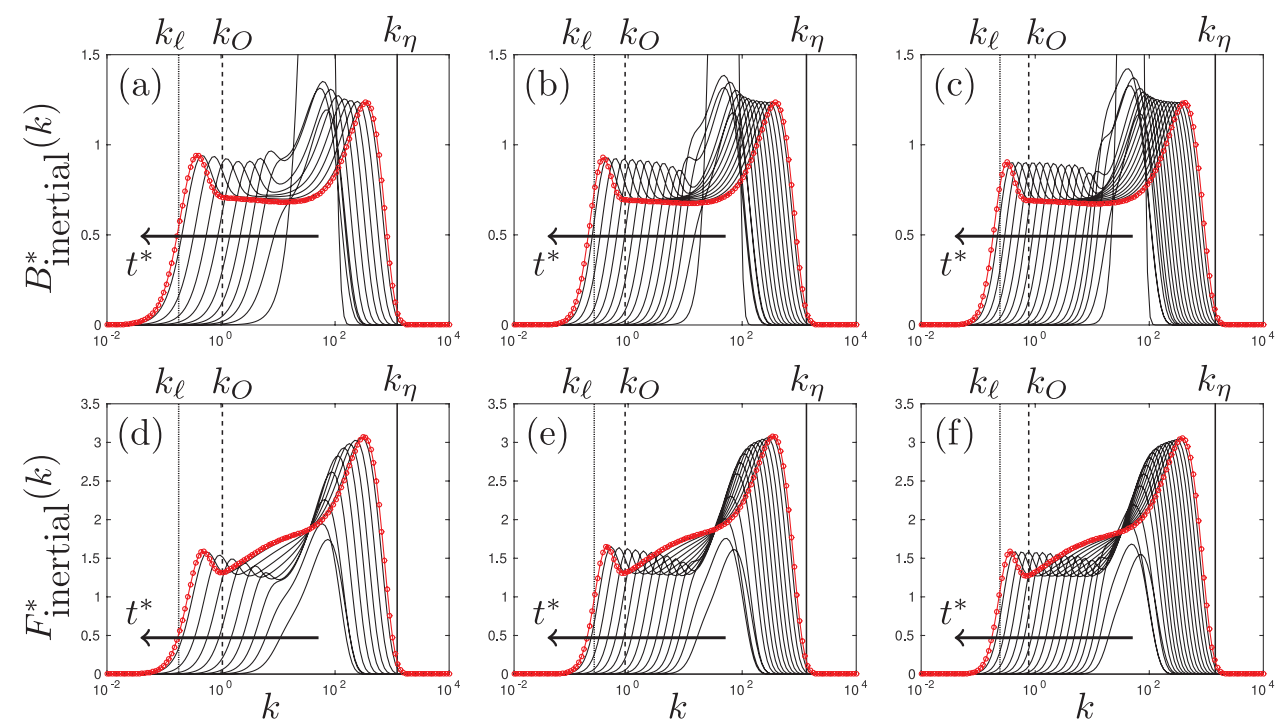

FIG. 8. Inertial range compensated spectra from Eqs. (5b) and (5c) for the buoyancy variance spectrum $B(k)$ : (a) $s=1$; (b) $s=3$; (c) $s=5$. Same for the co-spectrum of buoyancy-velocity $F(k)$ : (d) $s=1$; (e) $s=3$; (f) $s=5$. Times as in Figure 5, and the latest spectra are denoted with a heavy line and symbols. As in Figure 7, integral-, Ozmidov-, and Kolmogorov-length-scale-related wavenumbers $k_{\ell}, k_{O}$, and $k_{\eta}$ are indicated for the last spectra. 
within the chosen vertical range) are largely above the later time spectra, since dissipation is not fully built at the earliest times of computation. After a short transient during which the similarity shape evolves, the rescaled spectra develop three characteristic features. First, the inertial range zone corresponds to a plateau at intermediate wavenumbers, at a value slightly above the commonly admitted value $C_{K} \simeq 1.4$ for the Kolmogorov constant. This is consistent with isotropic EDQNM choosing $a_{0}=0.36$ in the eddy damping term. ${ }^{41}$ Second, the spectral region close to the integral scale wavenumber $\left(k_{\ell} \sim 1\right)$ exhibits a rather narrow peak different from the classical compensated spectrum in HIT. Indeed, as predicted for RT turbulence by Soulard ${ }^{42}$ a modification of the spectra near the integral scale at $k \sim 1$ is expected to scale as $k^{-3}$ in the inertial range zone delimited by the integral scale and the Ozmidov scale (with the corresponding Ozmidov wavenumber classically defined as $k_{O}=N^{3 / 2} / \varepsilon^{1 / 2}$ ). Note that strong differences between RT and USH turbulence prevail at large scales due to inhomogeneous specificities of RT. However, for smaller scales of the inertial range, it seems reasonable to seek same scaling laws between RT and USHT. Third, the observed bump at the upper end of the inertial region is the classically observed bottleneck in turbulence, from the depletion of non-linearity when reaching the dissipative range.

Rescaled buoyancy spectra, shown in Figures 8(a)-8(c) only for $s=1,3,5$, evolve towards a self-similar shape, as for the rescaled kinetic energy spectra: the inertial $k^{-5 / 3}$ scaling appears through a plateau at the Obukhov constant $C_{O} \simeq 0.7 .^{43}$ This value is consistent with the analysis of isotropic EDQNM for passive scalar with a single eddy damping term proposed by Ref. 44 . The plateau lies between the peak observed at the wavenumber $k_{\ell} \sim 1$ defined by the integral length scale, and the accumulation bottleneck between the end of the inertial subrange and the dissipative one. In $B_{\text {inertial }}^{*}(k)$, the latter bump is much more pronounced than in $E_{\text {inertial }}^{*}(k)$ in Figures 7(a)-7(f). This is also observed in passive scalar spectra in grid turbulence experiments. ${ }^{45}$

Finally, Figures 8(d)-8(f) show the velocity-buoyancy co-spectrum $F_{\text {inertial }}^{*}$ for the same $s=1,3$, 5. We observe a rapid drop in time of this rescaled flux from the initial conditions, before it settles to its self-similar shape. As for kinetic energy and buoyancy spectra, the inertial range is flanked by the peak at integral scale and the bottleneck. The inertial range seems to follow more loosely the $k^{-7 / 3}$ anticipated scaling, since, unlike $E_{\text {inertial }}^{*}$ and $B_{\text {inertial }}^{*}$, the convergence towards $k^{-7 / 3}$ is much slower, in terms of Reynolds number. (Lower Reynolds number runs, not presented here, also present this feature.) This may explain as well the difference of plateau level compared to Ref. 40 which is based on low Reynolds number Rayleigh-Taylor simulations. Moreover, the velocity-buoyancy flux is a cross-correlation statistics whose convergence is indeed expected to be slower than that of autocorrelation spectra. Finally, buoyancy acts at all ranges, and may disrupt the expected flux equilibrium in the inertial range. We recall that the energy cascade in this range is submitted to both the linear buoyancy force, and to the nonlinear triadic transfer.

\section{Anisotropy variation with $s$}

As mentioned, the dynamics of USH turbulence is strongly linked to the flow anisotropy which is produced by plumes from the effect of the buoyancy force. Hence, vertical kinetic energy is larger along the direction of gravity, along which the flow structures are elongated. Not only is the velocity field anisotropic, but also the density field, so that the description of their anisotropic structure requires specific quantities. We start in Sec. III D 1 by characterizing the anisotropy of the Reynolds stress tensor $\left\langle u_{i} u_{j}\right\rangle$ by computing its deviatoric part $b_{i j}=\left\langle u_{i} u_{j}\right\rangle /\left\langle u_{i} u_{i}\right\rangle-\delta_{i j} / 3, \delta_{i j}$ being the Kronecker tensor components. This provides a simple global view of the flow anisotropy at large scales. Then, we propose in Sec. III D 2 a refined description that permits a scale-by-scale evaluation of anisotropy, both for velocity and density fields, especially in the infrared subrange of interest.

\section{Anisotropy in the self-similar regime}

The first characterization of kinematic anisotropy is done with the anisotropy in the Reynolds stress components through its deviatoric tensor $\boldsymbol{b}$. From axisymmetry $\left(b_{11}=b_{22}\right)$ and incompressibility $\left(b_{i i}=0\right)$, only one component of $\boldsymbol{b}$ is required; we choose to compute $b_{33}=\left\langle u_{3} u_{3}\right\rangle /\left\langle u_{i} u_{i}\right\rangle-$ $1 / 3$, which is also dynamically dominant, since pertaining to the intensity of vertical structures 

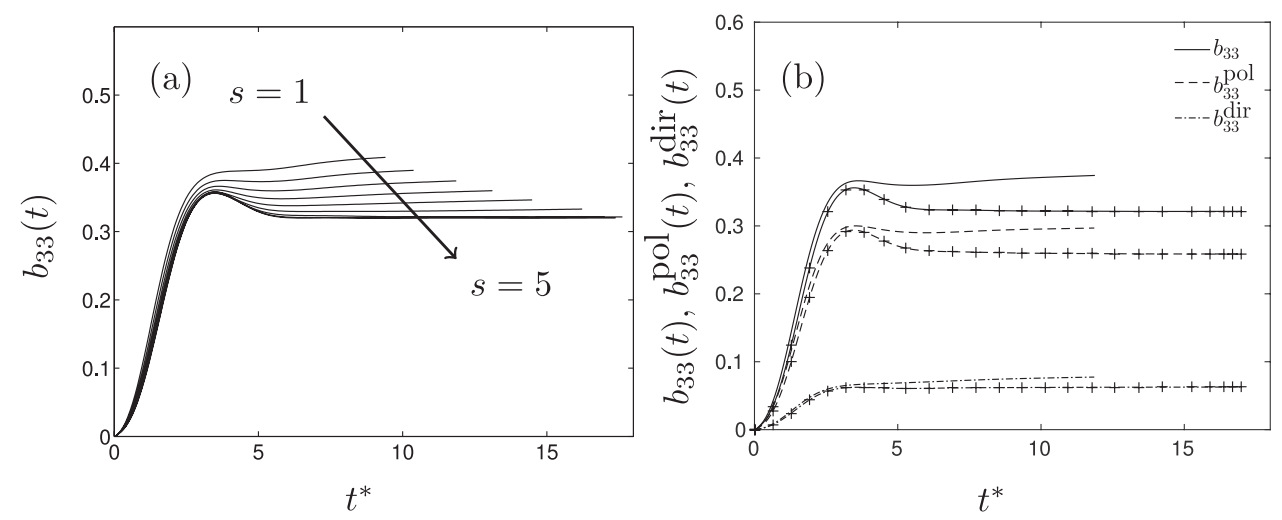

FIG. 9. One-point statistics of anisotropy: (a) vertical component of the Reynolds stress tensor anisotropy $b_{33}$ for the different infrared range power laws $k^{s}$, with $s$ from 1 to 5 ; (b) comparison for cases at $s=2$ and 4 (indicated by + ) between $b_{33}$ and its polarization and directivity components $b_{33}^{\text {pol }}$ and $b_{33}^{\text {dir. }}$.

developing in the flow. Its evolution in time is shown in Figure 9(a) for the different $s$ cases. The figure shows that, starting from $b_{33}=0$ for isotropic turbulence, $b_{33}$ grows rapidly and settles at an asymptotic value that depends on the infrared power law $s$ : between 0.42 for $s=1$ and 0.32 for $s=5$. The different levels of anisotropy in the self-similar regimes at different $s$ clearly show the dependence of the late-time structure of USH dynamics on initial conditions in the large scales. In mixing zones generated by the Rayleigh-Taylor instability, different levels of anisotropy as a function of the growth rate in the self-similar regime have been observed, see Gréa. ${ }^{46}$ This is consistent with what we show here, that it is possible to reach a range of final regimes by modifying the initial energy distribution at large scale.

As recalled in Sagaut and Cambon, ${ }^{29}$ the Reynolds stress tensor can be decomposed as $\left\langle u_{i} u_{j}\right\rangle=$ $\left\langle u_{i} u_{j}\right\rangle^{\text {iso }}+\left\langle u_{i} u_{j}\right\rangle^{\text {pol }}+\left\langle u_{i} u_{j}\right\rangle^{\text {dir }}$ with $\left\langle u_{i} u_{j}\right\rangle^{\text {iso }}$ the isotropic contribution over which the two anisotropic contributions are superimposed: $\left\langle u_{i} u_{j}\right\rangle^{\text {pol }}$ the polarization part and $\left\langle u_{i} u_{j}\right\rangle^{\text {dir }}$ the directionality part. Accordingly, $b_{33}$ can be split in two components as well: $b_{33}=b_{33}^{\text {pol }}+b_{33}^{\text {dir }}$ (logically $b_{33}^{\text {iso }}=0$ from the definition of $\left\langle u_{i} u_{j}\right\rangle^{\text {iso }}$ ). This decomposition permits to identify two sources of anisotropy coming from the structuring of the flow. First, the directionality contents testify of the accumulation of kinetic energy for wave vectors in the axial (i.e., along the axis of gravity) or the perpendicular direction. Second, the polarization part assesses the kind of structures that are produced once directional anisotropy has set in. For instance, an axisymmetric flow may contain mostly sheet-like (as in stably stratified turbulence ${ }^{31}$ ), jet-like structures (as in conducting fluid turbulence in a permanent background magnetic field ${ }^{27}$ ), or vortex-like ones (as in rotating turbulence ${ }^{26}$ ). In the USH case, the large directional anisotropy observed in Figure 9(b) attests a two-dimensional tendency. It can be shown that a purely two-dimensional flow settles an upper limit to the directional anisotropy at $b_{33}^{\mathrm{dir}}=1 / 6$. Furthermore, one also needs to examine the polarization in order to fully qualify the flow structure: it contains three components when $b_{33}^{\mathrm{pol}}=0$, but corresponds to an exactly two-dimensional two-component flow (vortex like) if $b_{33}^{\mathrm{pol}}=-1 / 3$. Values in between attest of preferential trends. On the contrary for RT or USH flows, the strong polarization anisotropy $\left(b_{33}^{\mathrm{pol}}=1 / 3\right)$ (see Figure 9) points out more precisely the dominance of vertical jet-like structures.

One expects that the polarization part represents $80 \%$ of the total velocity anisotropy in USH turbulence as from $\mathrm{RT}^{47}$ or previous USHT ${ }^{5}$ simulations. We confirm this with the plot of $b_{33}$ compared to $b_{33}^{\mathrm{pol}}$ and $b_{33}^{\mathrm{dir}}$ in Figure 9(b) for the cases $s=2$ (Saffman turbulence) and 4 (Batchelor turbulence). The asymptotic ratio $b_{33}^{\mathrm{pol}} / b_{33}$ indeed reaches $80 \%$ in both, even though $b_{33}$ does not asymptote exactly to the same value. This is observed in all the cases we computed independent of the large scales distribution. Figure 9(b) therefore shows that directional anisotropy is moderate compared to polarization anisotropy, but the value $\sim 0.3-0.4$ reached by their sum indicates an overall strongly anisotropic flow with preferential motion along the axis of gravity. We characterize further this anisotropy by examining its scale distribution in Sec. III D 2. 


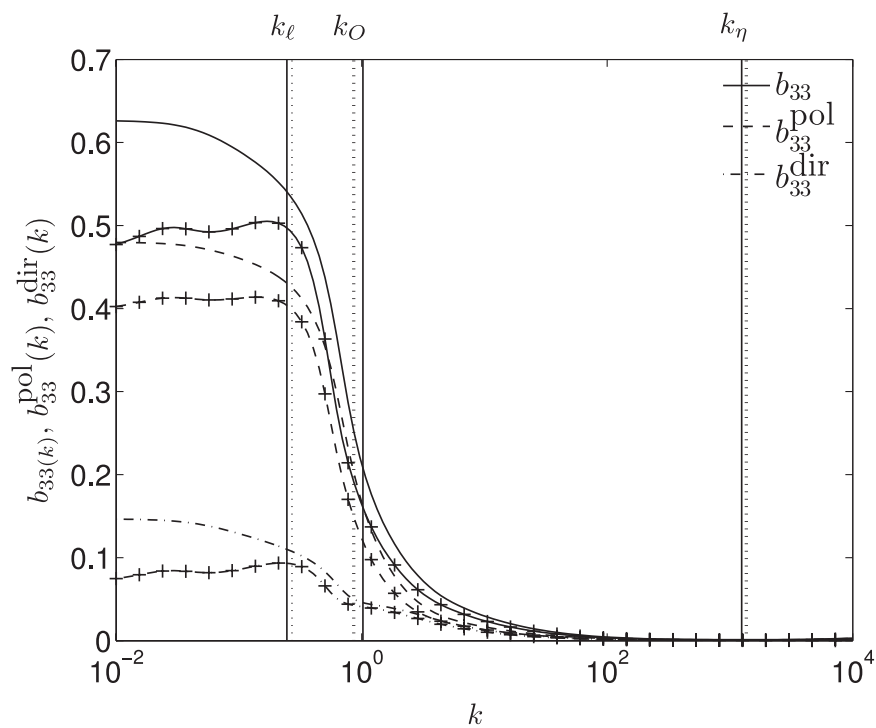

FIG. 10. Scale-by-scale deviatoric part $b_{33}(k)$ of the Reynolds stress tensor component $R_{33}=\left\langle\hat{u}_{3} \hat{u}_{3}\right\rangle$ and its polarization and directional contents $b_{33}^{\mathrm{pol}}(k)$ and $b_{33}^{\mathrm{dir}}(k)$ as a function of wavenumber $k$, for USH turbulence at $s=2$ and 4 (indicated by + ) for the same value of the Reynolds number $R e=10^{6}$. The vertical lines indicate wavenumbers corresponding to the integral, Ozmidov, and Kolmogorov length scales $k_{\ell}, k_{O}, k_{\eta}$, respectively (dashes for the case at $s=4$ ).

\section{Scale-by-scale anisotropy characterization}

We first investigate the anisotropy of $b_{33}$ scale-by-scale, by computing its value at each wavenumber $k$, and its polarization and directional contents. These are shown in Figure 10 for $s=2$ and 4. The figure first shows that maximal anisotropy $b_{33}(k) \simeq 0.5-0.6$ is reached in the large scales. It starts decreasing abruptly for wavenumbers larger than that corresponding to the integral length scale $k<k_{\ell}$-i.e., for structures smaller than the large energy-containing eddies-down to zero at the end of the inertial range, where isotropy is recovered.

From the polarization/directionality decomposition, one immediately observes that the largescale anisotropy is contributed by both large positive $b_{33}^{\mathrm{dir}}$ and $b_{33}^{\mathrm{pol}}$, as a trace of vertically elongated structures with a dominance of the vertical component of velocity as already mentioned in Sec. III D 1. The sharp drop of anisotropy, mostly due to a decrease of polarization anisotropy, attests of a structural change of turbulent eddies smaller than $\ell$ with a loss of the dominance of the vertical motions. Upon close observation of the similar, although slower, decrease of $b_{33}^{\mathrm{dir}}$, one also notes a change of the decay pace above the Ozmidov wavenumber $k_{O}$. This decrease of directional anisotropy corresponds to a recovery of three dimensional dependence of turbulent structures which can be seen as a second stage of the isotropization process down the turbulent scales.

The above analysis of anisotropy applies to a vector field such as velocity, but cannot be used for the density field which is scalar. In that case, one can introduce another measure of anisotropy, which consists in evaluating an angle $\gamma$ of "accumulation" of the given field about the axis of gravity, from the following weighted integral as proposed in Refs. 46 and 48:

$$
\sin ^{2} \gamma(k)=\frac{\int_{0}^{\pi} \mathcal{B}(k, \theta) \sin ^{3} \theta \mathrm{d} \theta}{\int_{0}^{\pi} \mathcal{B}(k, \theta) \sin \theta \mathrm{d} \theta}, \sin ^{2} \gamma=\frac{\int_{0}^{+\infty} k^{2} \int_{0}^{\pi} \mathcal{B}(k, \theta) \sin ^{3} \theta \mathrm{d} \theta \mathrm{d} k}{\int_{0}^{+\infty} k^{2} \int_{0}^{\pi} \mathcal{B}(k, \theta) \sin \theta \mathrm{d} \theta \mathrm{d} k} .
$$

This quantity gives the partition of anisotropy per wavenumber, but can also be integrated over $k$ to provide an average anisotropy for all scales. Values of $\sin ^{2} \gamma$ close to $2 / 3$ correspond to isotropic spectral distribution, whereas 1 indicates a complete concentration of $\mathcal{B}$ towards $\theta=\pi / 2$.

The distribution of the scale-integrated $\sin ^{2} \gamma$ plotted in Figure 11(a) shows a clear dependence of the scalar anisotropy with the flow initial conditions at large scale, or with $s$. It is larger for $s=1$ and decreases monotonously with $s$, down to about 0.75 in the similarity regime for $s \geq 4$. This seems to be an asymptote for this last case, and one expects also the same limitation of anisotropy in 

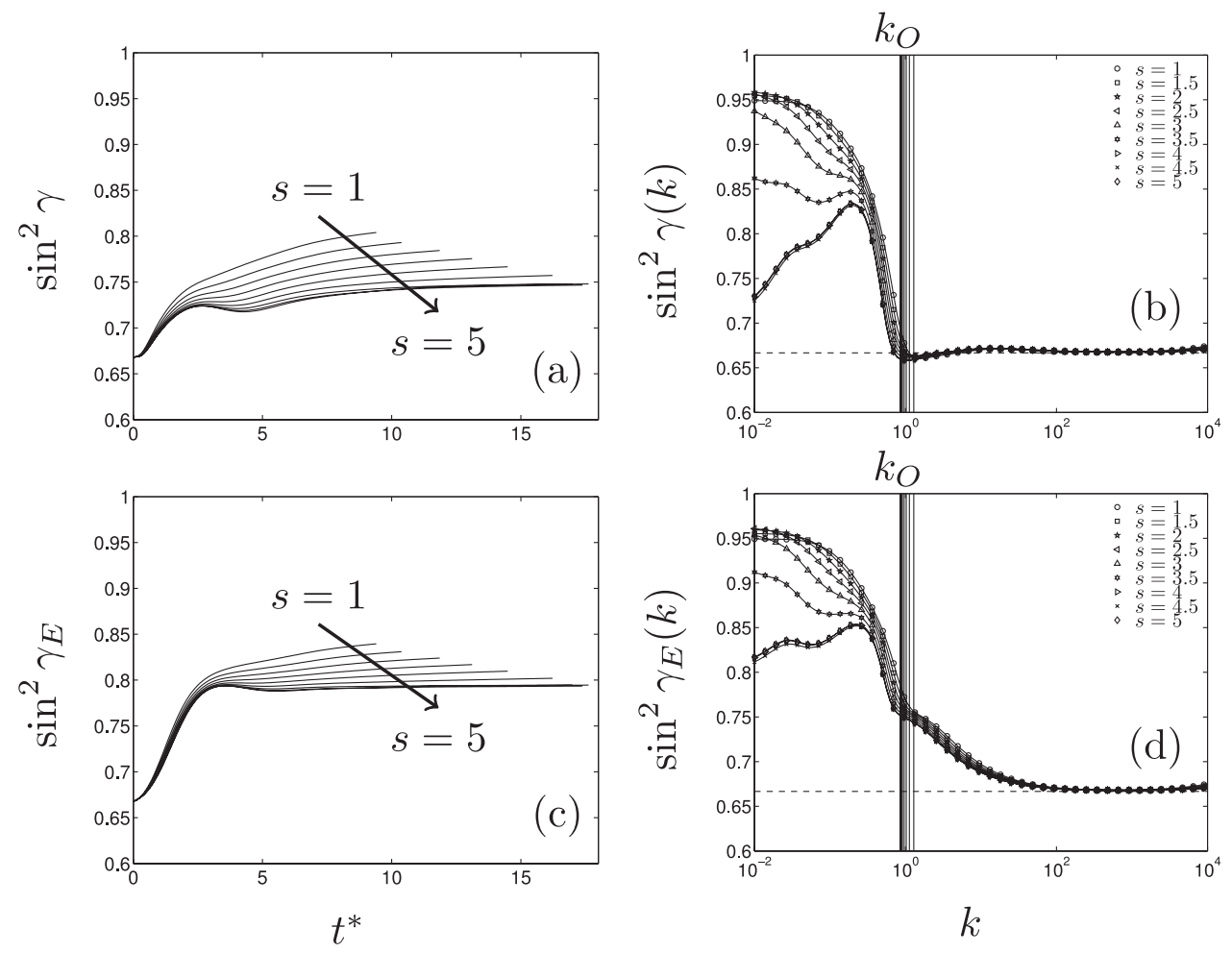

FIG. 11. (a) Evolution in time of the scale integrated $\sin ^{2} \gamma$ for cases $s=1$ to 5. (b) Distribution of $\sin ^{2} \gamma(k)$ (evaluated from Eq. (6)) as a function of wavenumber for the same cases, at $R e=10^{6}$. Vertical lines indicate the Ozmidov wavenumber for each case. Horizontal dashed line for the isotropic value. (c) Same as (a) for the scale integrated anisotropy of the kinetic energy spectrum $\sin ^{2} \gamma_{E}$. (d) Same as (b) for $\sin ^{2} \gamma_{E}(k)$.

the other cases, although the computations are not long enough to observe it (due to computational cost limitations).

The distribution of $\sin ^{2} \gamma(k)$ is shown on Figure 11(b) at the same Reynolds number $R e=10^{6}$ reached by all the $s$ runs at different times. The decomposition of anisotropy per wavenumber in Figure 11(b) shows different tendencies for different spectral subranges. First, at large scale from $k \sim 10^{-2}$ to $k \sim 1$, we can distinguish two trends, for cases $s \in[1,3]$, on the one hand, and cases $s \geq 4$ on the other. The first set of flows contains larger anisotropy in the energy-containing subrange, since $\sin ^{2} \gamma$ is close to unity. Their lowest wavenumbers have maximal anisotropy. The second group shows a completely different behavior: at lowest wavenumber, $\sin ^{2} \gamma \simeq 0.7$, meaning lower anisotropy. Moreover, $\sin ^{2} \gamma(k)$ increases from $k=10^{-2}$ to $k \sim 1$. These two different large-scale dynamics are linked to the infrared slope. While the $s \geq 4$ runs are characterized by a steeper infrared range implying the presence of backscatter that damps anisotropy, the lack of backscatter in the energy cascade when $s \in[1,3]$ allows the full development of anisotropy in the large scales under the effect of the buoyancy force. Also, nonlinear terms are acting on the largest scales and tend to scramble them. It leads to a decorrelation at low wavenumbers which limits the amount of large scale anisotropy. A value of $s$ between 3 and 4 that appeared in the dynamics of isotropic turbulence $(s=3.45)^{13}$ therefore also seems to be pivotal for the development of anisotropy in the infrared subrange of USHT.

Figure 11(b) also shows that differences in the level of anisotropy between different $s$ cases are completely removed in scales smaller than the integral length scales, or, in the plots, for wavenumbers $k$ larger than about 1 . From this wavenumber, the anisotropy of $\mathcal{B}$ quickly drops to 0.66 in all runs, indicative of isotropy of the buoyancy field at these scales, and plateaus at this value throughout the inertial subrange and down to the dissipative scales.

Finally, we also evaluate the integrated anisotropy $\sin ^{2} \gamma_{E}$ and its scale-by-scale distribution $\sin ^{2} \gamma_{E}(k)$ for the velocity field from Eq. (6) by replacing the buoyancy variance spectrum $\mathcal{B}(k, \theta)$ 
by the kinetic energy spectrum $\mathcal{E}(k, \theta)$ in the integral. Their evolutions and distributions are plotted in Figures 11(c) and 11(d) for the different values of $s$. Figure 11(c) shows that the time evolution of $\sin ^{2} \gamma_{E}$ is similar to that of $\sin ^{2} \gamma$, but reaches a plateau faster and at a slightly higher level of anisotropy. The overall distribution of velocity anisotropy $\sin ^{2} \gamma_{E}(k)$ in Figure 11(d) is similar to that of buoyancy anisotropy of Figure 11(b): the large-scale anisotropy is either increasing or decreasing with $k$ in the infrared subrange for $s$ smaller or larger than 4; isotropy is recovered in the dissipative subrange; the plateau in the inertial subrange is not as large as for buoyancy anisotropy, since the transition from strongly anisotropic infrared subrange to mostly isotropic inertial dynamics is not as sharp. The separation wavenumber between two such ranges can be estimated by the Ozmidov wavenumber $k_{O}=N^{3 / 2} \varepsilon^{-1 / 2}$, where $\varepsilon$ is the kinetic energy dissipation. ${ }^{49} k_{O}$ is shown on both Figures 11(b) and 11(d). It appears to mark clearly the beginning of the isotropic range in buoyancy, or the limit of the trend towards isotropy recovery in velocity. One should, however, beware of the fact that in our computations dissipation varies weakly from one case to another and that stratification intensity $N$ is the same for all runs, so that the Ozmidov wavenumber does not vary much. Additional parametric studies would be required to confirm its universal role in separating anisotropic from isotropic scales.

\section{Detailed anisotropic spectra}

We further analyse here the detailed spectral distribution first of the vertical velocity autocorrelation spectrum $R_{33}(k)$, and the angular velocity-buoyancy flux distribution $\mathcal{F}(k, \theta)$. For both these quantities, scaling laws have been proposed.

Soulard and Griffond ${ }^{40}$ propose a supplemented anisotropic correction due to buoyancy to the isotropic contribution in $R_{33}(k)$ which scales as $k^{-3}$ from linear response theory (see the Appendix). This scaling should apply for wavenumbers smaller than $k_{O}$ and approaching the integral length scale. Moreover, one expects that, in the inertial range at larger wavenumbers, Kolmogorov scaling $k^{-5 / 3}$ should be recovered (see also Burlot et al. ${ }^{6}$ ). This last scaling is clearly recovered in the $R_{33}(k)$ spectrum of Figure 12. When applying the isotropic-directional-polarization decomposition mentioned in Sec. III D 1, we observe that the $-5 / 3$ power law in the inertial range of $R_{33}(k)$ is entirely contributed by its isotropic component $R_{33}^{\text {iso }}(k)$ in the decomposition. The two other anisotropic parts $R_{33}^{\mathrm{dir}}(k)$ and $R_{33}^{\mathrm{pol}}(k)$ are negligible in this range. They, however, are observed to scale as $k^{-3}$ (at least not too far from it) from the peak wavenumber down to the small scales, so that they both are at the origin of the $k^{-3}$ narrow subrange, with a preferential contribution of the polarization part which is significantly larger than the directional part throughout the large scale range.

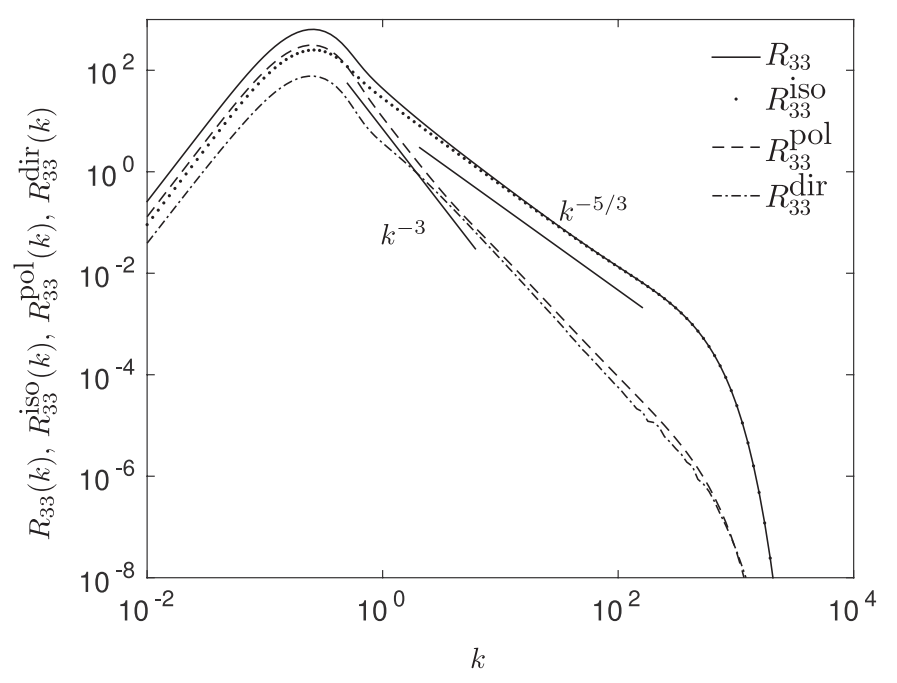

FIG. 12. Spectrum $R_{33}(k)$ of the two-point correlation function of vertical velocity with vertical separation $\left\langle u_{3}(\mathbf{x}) u_{3}(\mathbf{x}+\right.$ $\left.\left.r \mathbf{e}_{z}\right)\right\rangle$, and its directional and polarization components, for $s=3$ at $\operatorname{Re}=10^{6}$. 

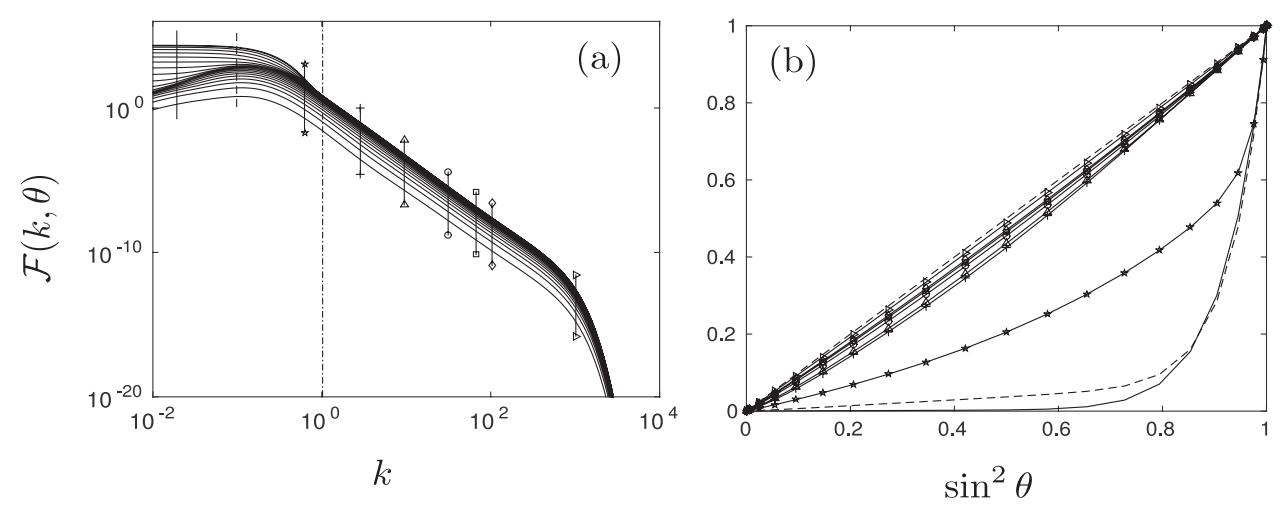

FIG. 13. Distribution of buoyancy flux $\mathcal{F}(k, \theta)$ for the $s=2$ case: (a) $k$-dependence at different given angles $\theta$; (b) dependence with $\sin ^{2} \theta$ at the given wavenumbers $k=0.02,0.1,0.6,3,10,30,70,100$, and 1000 shown in panel (a).

Regarding the explicit angular dependence of the spectra, a functional law in $\theta$ is hard to come by for anisotropic spectra in general. Kaneda and Yoshida ${ }^{50}$ developed the deviation spectra of stably stratified turbulence in powers of the stratification parameter, and thus proposed that the vertical velocity-buoyancy correlation spectrum depends angularly on $\theta$ as $\mathcal{F}(k, \theta) \simeq \sin ^{2} \theta$ in the inertial range. These authors used DNS data to check the validity of this dependence, but DNS statistics are very noisy, even at high resolution. The EDQNM approach is well suited for this since the computed spectra are explicit functions of $k$ and $\theta$. We therefore plot in Figure 13(a) the $k$-dependent $\mathcal{F}(k, \theta)$ for a series of orientations $\theta$, and in Figure 13(b) its dependence on $\sin ^{2} \theta$ for different wavenumbers $k$. Figure 13(a) shows that the flux is larger at $\theta \simeq \pi / 2$ whatever the wavenumber, so that the effect of the buoyancy force is felt at all scales. Of course, it is more intense in the energy containing range, at very small wavenumbers, that is, at the largest scales in the flow. Figure 13(b) is a partial check of the $\theta$-dependence proposed by Kaneda and Yoshida. At wavenumbers $k$ in the inertial range, $\mathcal{F}(k, \theta)$ is clearly linear in $\sin ^{2} \theta$, thus confirming the validity of the proposed scaling. Note that we have also verified the different angular dependences for $\mathcal{R}_{33}$ following the law proposed in the Appendix in the inertial range. For larger or smaller wavenumbers, the scaling does not apply at all.

However, our results show some noticeable differences with the theory proposed by Kaneda and Yoshida ${ }^{50}$ and Soulard and Griffond ${ }^{40}$ The return to isotropy at wavenumbers larger than $k_{O}$ is much sharper on the scalar spectra $\mathcal{B}(k, \theta)$ compared to the velocity spectra $\mathcal{E}(k, \theta)$ as shown in Figure 11(b). This has been also observed in RT DNS. ${ }^{40}$ As a consequence, no perceptible directional anisotropy can be detected in the inertial range of $\mathcal{B}$ while a $\sin ^{2} \theta$ dependence is expected from the theory. The fact that EDQNM is able to reproduce this feature suggests the importance of non-local effects in the transfer which are not taken into account in theories such as Ref. 50 or models such as Ref. 30. This aspect is also confirmed when comparing the different constants for the expected scaling law from Ref. 40 and extracted from our EDQNM simulations as detailed in the Appendix.

\section{CONCLUSION}

In this work, a numerical study of unstably stratified homogeneous turbulence was performed using an eddy-damped quasi-normal Markovian model. This model was developed and its prediction compared to results from direct numerical simulations by Burlot et al., ${ }^{6}$ showing very good agreement. The model gives access to unprecedented parametric studies at high Reynolds number, and is free from box-size confinement as in quickly time-evolving turbulence, either decaying, or with increasing energy as in USH turbulence. Of course, at very large Reynolds number, the time step reduction still limits somehow the duration of the computations, but this concerns huge values of $\operatorname{Re} \sim O\left(10^{6}\right)$. 
We use here the EDQNM model to explore the influence of energy distribution of the initial spectral densities of velocity auto-correlation and buoyancy auto-correlation on the late-time dynamics. The slope $s$ of the infrared spectrum, defined as $\lim _{k \rightarrow 0} E(k, t) \rightarrow k^{s}$, was the parameter used to change this energy distribution. Its value varies from $s=1$ to $s=5$ with a step of 0.5 (not all the intermediate values were presented here). The simulations were pursued in time to sufficiently high Reynolds numbers so that a self-similar regime is reached. The characteristics of the various self-similar final regimes thus reached were analysed in detail.

We found that the infrared slope has a clear and non-negligible impact on the late-time dynamics as suggested by Ref. 3. First, it influences the ratio between stratification effects and nonlinear effects (Figure 1(b)) as well as the partition between kinetic and buoyancy variance (Figure 1(c)). Also the anisotropy of the flow is impacted by the initial condition. Both polarization and directionality show variations of late time dynamics with the modification of initial $s$ (Figs. 9(a) and 9(b)). This indicates that turbulent structures developing in buoyancy driven flows can be dependent on the initial condition even after a long time of the flow evolution.

Energy spectra also show a change of distribution of large scale energy due to backscatter effects. This was already known in homogeneous isotropic turbulence, ${ }^{13}$ but we clearly identified it here for different values of $s$ for USH turbulence. We observe a transition range for $s \in[3,4]$ in our strongly anisotropic case, consistent with the value $s=3.45$ found in isotropic turbulence. Large-scale compensated spectra (Figures 5 and 6 ) indicate a non-permanent evolution of large scale for $s \geq 3.5$. Furthermore, inertial range compensated spectra indicate also a modification of the self-similar spectral shape for kinetic energy by the emergence of a peak on the lower bound of the inertial range, near the integral scale. This was first identified by Soulard using DNS and modelling. ${ }^{40}$

We also characterize finely the scalings of the spectra in $k$ and $\theta$, predicted by theory based on strong assumptions: the $k^{-3}$ inertial subrange is observed in our results thanks to the very high Reynolds number, and we further find that it is essentially due to polarization anisotropy; the $\sin ^{2} \theta$ dependence of the velocity-buoyancy correlation spectrum in the inertial range appears also very clearly in our results, thanks to the EDQNM model, with respect to more noisy DNS data. We also obtain close to $k^{-7 / 3}$ scaling in compensated spectra, but we show that the convergence to this power law in terms of Reynolds number is very slow: $R e \simeq 10^{6}$ is required to observe a tendency. However, the angular dependence on the buoyancy spectrum is not recovered suggesting limitation of the local theory proposed by Ref. 40. A possible explanation can be the importance of non-local effects in the transfer.

Overall, our results bring new information on the permanence of the big eddies that initially drive the flow dynamics in USH turbulence, and thus on the essential role played by initial conditions. In this work, our model assumes that the stratification profile does not vary in time, or very slowly with respect to turbulence time scales. This may not be exact in turbulent mixing or convective zones, in which turbulence interacts with the background density fields and modifies its mean variation, so that an extension of our analysis to time-varying stratification intensity $N$ is called for as in Refs. 3 and 5.

\section{APPENDIX: COMPARISONS WITH EXPECTED SCALING IN THE INERTIAL RANGE FROM LOCAL THEORY ${ }^{30,40}$}

The scaling laws for the RT inertial range have been investigated by Soulard and Griffond ${ }^{40}$ using the spectral model of Canuto et al. ${ }^{30}$ and applying the spectral equilibrium method (or linear response theory) introduced by Kaneda and Yoshida. ${ }^{50}$ The basic idea consists in assuming at leading order a Kolmogorov-Obukhov $k^{-5 / 3}$ isotropic spectrum and then in adding a small correction in order to take into account stratification effects. While the inertial forces are assumed dominant at those scales, the corrective terms in the spectra are used to keep balance with the presence of the buoyancy force. The expressions derived from this procedure, which are applied to the Canuto et al. model, give for the vertical Reynolds stress spectra $\mathcal{R}_{33}(k, \theta)$, the buoyancy spectrum $\mathcal{B}(k, \theta)$, and 
TABLE III. Values of the different constants appearing in scaling law Eqs. (A1a)-(A1c) for the inertial zone.

\begin{tabular}{lccccc}
\hline \hline & $C_{K}$ & $C_{O}$ & $C_{e}$ & $C_{f}$ & $C_{p}$ \\
\hline Proposed by 40 & 1.9 & 1.71 & 1.23 & 1.08 & 1.10 \\
Evaluated from & 1.4 & 0.7 & {$[1.6,2.5]$} & {$[2,3]$} & {$[-0.06,0.04]$} \\
EDQNM simulations & & & & & \\
\hline \hline
\end{tabular}

the vertical buoyancy flux $\mathcal{F}(k, \theta)$ the expressions summarized below,

$$
\begin{aligned}
& \mathcal{R}_{33}(k, \theta)=\mathcal{R}_{33}^{(i s o)}(k, \theta)+\mathcal{R}_{33}^{(p o l)}(k, \theta)+\mathcal{R}_{33}^{(\text {dir })}(k, \theta) \\
& \text { with } \quad \mathcal{R}_{33}^{(i s o)}(k, \theta)=\left(\frac{1}{4 \pi} C_{K} \varepsilon^{2 / 3} k^{-11 / 3}-\frac{5}{16} N^{2} \frac{1}{4 \pi} C_{e}\left(C_{K}+C_{O} \varepsilon_{B} \varepsilon^{-1}\right) k^{-5}\right) \sin ^{2} \theta \\
& \mathcal{R}_{33}^{(d i r)}(k, \theta)=N^{2} \frac{1}{4 \pi} C_{e}\left(C_{K}+C_{O} \varepsilon_{B} \varepsilon^{-1}\right) k^{-5}\left(\sin ^{2} \theta-\frac{2}{3}\right) \sin ^{2} \theta \\
& \mathcal{R}_{33}^{(p o l)}(k, \theta)=N^{2} \frac{1}{4 \pi} C_{e}\left(C_{K}+C_{O} \varepsilon_{B} \varepsilon^{-1}\right) k^{-5} \sin ^{4} \theta \\
& \mathcal{B}(k, \theta)=\frac{1}{4 \pi} C_{O} \varepsilon_{B} \varepsilon^{-1 / 3} k^{-11 / 3}-C_{p} N^{2} \frac{1}{4 \pi}\left(C_{K}+C_{O} \varepsilon_{B} \varepsilon^{-1}\right)\left(3-2 \sin ^{2} \theta-\frac{25}{12} \varepsilon_{B} \varepsilon^{-1}\right) k^{-5} \\
& \mathcal{F}(k, \theta)=C_{f} N \frac{1}{4 \pi}\left(C_{K} \varepsilon^{1 / 3}+C_{O} \varepsilon_{B} \varepsilon^{-2 / 3}\right) k^{-11 / 3} \sin ^{2} \theta
\end{aligned}
$$

In Eqs. (A1a)-(A1c), one recognizes the main isotropic scaling in $k^{-11 / 3}$ for $\mathcal{R}_{33}$ and $\mathcal{B}$ and an anisotropic deviation proportional to $N^{2}$ in $k^{-5}$. This gives spherically integrated spectra evolving as $k^{-5 / 3}$ with an added $k^{-3}$ contribution from stratification. For the vertical flux $\mathcal{F}$, the leading term is $k^{-11 / 3}$ giving the classical $k^{-7 / 3}$ law on integrated spectra proposed by Ref. 39 .

The Kolmogorov and Obukhov constants $C_{K}$ and $C_{O}$ are then supplemented by three additional constants $C_{e}, C_{f}$, and $C_{p}$ for the anisotropic parts of the spectra. We propose to compare the values for these constants between those suggested by Ref. 40 for RT and those measured from our USHT EDQNM simulations assuming the validity of Eqs. (A1a)-(A1c). The results are presented in Table III. To begin with, it can be noticed that the values for the Kolmogorov and Obukhov constants $C_{K}$ and $C_{O}$ are relatively different. This can be explained as the constants in Soulard and Griffond ${ }^{40}$ have been extracted from DNS with relatively low turbulent Reynolds number $(R e \sim 800)$. On the contrary, the values from EDQNM simulations are more coherent with existing data such as Champagne et al ${ }^{51}$ Then considering the values for $C_{e}, C_{f}$, and $C_{p}$, we are not able to find accurate values from our EDQNM simulations since compensated spectra do not lead to clear plateau despite the large value of the Reynolds number. This is clearly observed, for instance, in Figure 8 for $F^{*}$ which allows an estimate of $2 C_{f} / 3$. Taking into account the discrepancies on $C_{K}$ and $C_{O}$ between EDQNM and Soulard and Griffond, ${ }^{40}$ the estimated values for $C_{e}$ and $C_{f}$ are coherent. However, the constant $C_{p}$ is very different and expresses the strong return to isotropy for scales smaller than Ozmidov on the scalar spectra in EDQNM simulations and also in DNS of Soulard and Griffond. ${ }^{40}$ This is the evidence of a limitation in the theory proposed by Soulard and Griffond ${ }^{40}$ which may be explained by the importance of non-local interactions in the equilibrium range taken into account by the EDQNM model but not by Ref. 30.

\footnotetext{
${ }^{1} \mathrm{~J}$. Lindl, "Development of the indirect-drive approach to inertial confinement fusion and the target physics basis for ignition and gain," Phys. Plasmas 2, 3933 (1995).

${ }^{2}$ G. Dimonte, D. L. Youngs, A. Dimits, S. Weber, M. Marinak, S. Wunsch, C. Garasi, A. Robinson, M. J. Andrews, P. Ramaprabhu et al., "A comparative study of the turbulent Rayleigh-Taylor instability using high-resolution threedimensional numerical simulations: The Alpha-Group collaboration," Phys. Fluids 16, 1668 (2004).

${ }^{3}$ O. Soulard, J. Griffond, and B.-J. Gréa, "Large-scale analysis of self-similar unstably stratified homogeneous turbulence," Phys. Fluids 26, 32 (2014).
} 
${ }^{4}$ J. Griffond, B. J. Gréa, and O. Soulard, "Unstably stratified homogeneous turbulence as a tool for turbulent mixing modeling,” J. Fluids Eng. 136, 091201 (2014).

5 J. Griffond, B.-J. Gréa, and O. Soulard, "Numerical investigation of self-similar unstably stratified homogeneous turbulence," J. Turbul. 16, 167 (2015).

${ }^{6}$ A. Burlot, B.-J. Gréa, F. S. Godeferd, C. Cambon, and J. Griffond, "Spectral modelling of high Reynolds number unstably stratified homogeneous turbulence," J. Fluid Mech. 765, 17 (2015).

${ }^{7}$ S. T. Thoroddsen, C. W. Van Atta, and J. S. Yampolsky, "Experiments on homogeneous turbulence in an unstably stratified fluid," Phys. Fluids 10, 3155 (1998).

${ }^{8}$ G. K. Batchelor, V. M. Canuto, and J. R. Chasnov, "Homogeneous buoyancy-generated turbulence," J. Fluid Mech. 235, 349 (1992).

${ }^{9}$ D. Livescu and J. R. Ristorcelli, “Buoyancy-driven variable-density turbulence,” J. Fluid Mech. 591, 43 (2007).

${ }^{10}$ D. Chung and D. I. Pullin, "Direct numerical simulation and large-eddy simulation of stationary buoyancy-driven turbulence," J. Fluid Mech. 643, 279 (2010).

${ }^{11}$ J. R. Ristorcelli and T. T. Clark, "Rayleigh-Taylor turbulence: Self-similar analysis and direct numerical simulations," J. Fluid Mech. 507, 213 (2004).

12 O. Poujade and M. Peybernes, "Growth rate of Rayleigh-Taylor turbulent mixing layers with the foliation approach," Phys. Rev. E 81, 016316 (8 pages) (2010).

${ }^{13}$ M. Lesieur and S. Ossia, "3D isotropic turbulence at very high Reynolds numbers: EDQNM study," J. Turbul. 1, 1 (2000).

${ }^{14}$ M. Meldi and P. Sagaut, "On non-self-similar regimes in homogeneous isotropic turbulence decay," J. Fluid Mech. 711, 364 (2012).

15 G. K. Batchelor, “The role of big eddies in homogeneous turbulence," Proc. R. Soc. A 195, 513 (1949).

${ }^{16}$ P. Saffman, "New results on the statistical properties of the large eddies in homogeneous turbulence," Phys. Fluids 10, S301 (1967).

${ }^{17}$ P. Saffman, “The large-scale structure of homogeneous turbulence," J. Fluid Mech. 27, 581 (1967).

18 T. Teitelbaum and P. D. Mininni, "Decay of Batchelor and Saffman rotating turbulence," Phys. Rev. E 86, 066320 (13 pages) (2012).

${ }^{19}$ P. A. Davidson, "The decay of Saffman and Batchelor turbulence subject to rotation, stratification or an imposed magnetic field,” J. Phys.: Conf. Ser. 318, 072025 (8 pages) (2011).

${ }^{20}$ M. Meldi and P. Sagaut, "Further insights into self-similarity and self-preservation in freely decaying isotropic turbulence," J. Turbul. 14, 24 (2013).

${ }^{21}$ W. H. Cabot and A. W. Cook, "Reynolds number effects on Rayleigh-Taylor instability with possible implications for type Ia supernovae," Nat. Phys. 2, 562 (2006).

${ }^{22}$ R. H. Kraichnan, "Inertial-range transfer in two- and three-dimensional turbulence," J. Fluid Mech. 47, 525 (1971).

${ }^{23}$ S. A. Orszag, "Analytical theories of turbulence," J. Fluid Mech. 41, 363 (1969).

${ }^{24}$ S. A. Orszag, in Les Houches Summer School in Physics 1973, edited by R. Balian and J.-L. Peabe (Gordon and Breach, New York, 1977), pp. 227-374.

${ }^{25}$ F. S. Godeferd and C. Staquet, "Statistical modelling and direct numerical simulations of decaying stably stratified turbulence. Part 2. Large-scale and small-scale anisotropy," J. Fluid Mech. 486, 115 (2003).

${ }^{26}$ C. Cambon, N. N. Mansour, and F. S. Godeferd, “Energy transfer in rotating turbulence,” J. Fluid Mech. 337, 303 (1997).

27 B. Favier, F. S. Godeferd, C. Cambon, A. Delache, and W. J. T. Bos, "Quasi-static magnetohydrodynamic turbulence at high Reynolds number," J. Fluid Mech. 681, 434 (2011).

${ }^{28}$ R. S. Rogallo, Technical Report 81315, NASA, memo, 1981.

29 P. Sagaut and C. Cambon, Homogeneous Turbulence Dynamics (Cambridge University Press, 2008), ISBN 9780521855488, URL http://books.google.fr/books?id=zo1QIn51S5IC.

${ }^{30}$ V. M. Canuto, M. S. Dubovikov, and A. Dienstfrey, "A dynamical model for turbulence. IV. Buoyancy-driven flows," Phys. Fluids 9, 2118 (1997).

${ }^{31}$ F. S. Godeferd and C. Cambon, "Detailed investigation of energy transfers in homogeneous stratified turbulence," Phys. Fluids 6, 2084 (1994).

32 S. A. Orszag and G. S. Patterson, "Numerical simulation of three-dimensional homogeneous isotropic turbulence," Phys. Rev. Lett. 28, 76 (1972).

${ }^{33}$ M. Lesieur, Turbulence in Fluids, Fluid Mechanics and Its Applications Series (Springer, 2008), ISBN 9781402064357, URL http://books.google.fr/books?id=xKUDN22Y7OYC.

34 T. von Kármán and C. Lin, "On the concept of similarity in the theory of isotropic turbulence," Rev. Mod. Phys. 21, 516 (1949).

${ }^{35}$ V. Borue and S. A. Orszag, "Self-similar decay of three-dimensional homogeneous turbulence with hyperviscosity," Phys. Rev. E 51, R856 (1995).

${ }^{36}$ S. B. Pope, Turbulent Flows (Cambridge University Press, 2000), ISBN 9780521598866, URL http://books.google.fr/ books?id=HZsTw9SMx-0C.

37 J. L. Lumley, "The spectrum of nearly inertial turbulence in a stably stratified fluid," J. Atmos. Sci. 21, 99 (1964).

${ }^{38}$ K. Nagata, Y. Sakai, S. Komori, and T. Kubo, "Velocity and passive scalar cross spectra in stably and unstably stratified grid turbulence," J. Fluid Sci. Technol. 2, 109 (2007).

39 J. L. Lumley, "Similarity and the turbulent energy spectrum," Phys. Fluids 10, 855 (1967).

${ }^{40}$ O. Soulard and J. Griffond, "Inertial-range anisotropy in Rayleigh-Taylor turbulence," Phys. Fluids 24, 025101 (2012).

${ }^{41}$ J. C. André and M. Lesieur, "Influence of helicity on the evolution of isotropic turbulence at high Reynolds number," J. Fluid Mech. 81, 187 (1977).

42 O. Soulard, "Implications of the Monin-Yaglom relation for Rayleigh-Taylor turbulence," Phys. Rev. Lett. 109, 254501 (2012).

${ }^{43}$ A. M. Obukhov, "The structure of the temperature field in a turbulent flow," Izv. Akad. Nauk. SSSR, Ser. Geogr. Geophys. 13, 58 (1949). 
${ }^{44}$ J. R. Herring, D. Schertzer, M. Lesieur, G. R. Newman, J. P. Chollet, and M. Larcheveque, "A comparative assessment of spectral closures as applied to passive scalar diffusion," J. Fluid Mech. 124, 411 (1982).

${ }^{45}$ L. Mydlarski and W. Warhaft, "Passive scalar statistics in High-Peclet-number grid turbulence," J. Fluid Mech. 358, 135 (1998).

${ }^{46}$ B.-J. Gréa, "The rapid acceleration model and the growth rate of a turbulent mixing zone induced by Rayleigh-Taylor instability," Phys. Fluids 25, 015118 (20 pages) (2013).

${ }^{47}$ B.-J. Gréa, O. Soulard, and J. Griffond, personal communication, IWPCTM conference (2010).

${ }^{48}$ C. Cambon and B.-J. Gréa, "The role of directionality on the structure and dynamics of strongly anisotropic turbulent flows," J. Turbul. 14, 50 (2013).

${ }^{49}$ R. Ozmidov, "On energy distribution over multi-scale motions of ocean water," Izv., Acad. Sci., USSR, Atmos. Oceanic Phys. 4, 257 (1965).

${ }^{50}$ Y. Kaneda and K. Yoshida, "Small-scale anisotropy in stably stratified turbulence," New J. Phys. 6, 34 (2004).

${ }^{51}$ F. H. Champagne, C. A. Friehe, J. C. LaRue, and J. C. Wynagaard, "Flux measurements, flux estimation techniques, and fine-scale turbulence measurements in the unstable surface layer over land," J. Atmos. Sci. 34, 515 (1977). 